\title{
Big Data-aware News Recommendation System According to Regional Twitter Users' Interests
}

\section{Maryam Bagheri}

University of Mohaghegh Ardabili Faculty of Engineering

\section{Shahram Jamali}

University of Mohaghegh Ardabili Faculty of Engineering

Reza Fotohi ( $\square$ fotohi.reza@gmail.com )

Shahid Beheshti University https://orcid.org/0000-0002-1848-0220

\section{Research Article}

Keywords: Big data, News recommendation system, Social networks, Spark framework, Twitter

Posted Date: September 13th, 2021

DOI: https://doi.org/10.21203/rs.3.rs-392181/v1

License: (1) This work is licensed under a Creative Commons Attribution 4.0 International License. Read Full License 


\title{
Big Data-aware News Recommendation System According to Regional Twitter Users' Interests
}

\author{
Maryam Bagheri $^{1}$. Shahram jamali ${ }^{1,2}$ (D) Reza Fotohi $^{3 *}$
}

\begin{abstract}
Nowadays with the development of technology and access to the Internet everywhere for everyone, the interest to get the news from newspapers and other traditional media is decreasing. Therefore, the popularity of news websites is ascending as the newspapers are changing into electronic versions. News websites can be accessed from anywhere, i.e., any country, city, region, etc. So, the need to present the news depends on where the reader is from can be a research area, as with facing with variety of news topics on websites readers prefer to choose those which more often show the news, they are interested in on their home pages. Based on this idea we represent the technique to find favorite topics of Twitter users of certain geographical districts to provide news websites a way of increasing popularity. In this work we processed tweets. It seems that tweets are some small data, but we found out that processing this small data needs a lot of time, due to the repetition of the algorithm a lot and many searches to be done. Therefore, we categorized our work as big data. To help this problem we developed our work in the Spark framework. Our technique includes 2 phases; Feature Extraction Phase and Topic Discovery Phase. Our analysis shows that with this technique we can get the accuracy between $68 \%$ and $76 \%$, in 3 developments 3-fold, 5-fold, and 10-fold.
\end{abstract}

Keywords Big data . News recommendation system . Social networks . Spark framework . Twitter

Maryam Bagheri

Maryambagheri@live.com

Shahram jamali

Jamali@uma.ac.ir

$\triangle$ Reza Fotohi*

$\underline{\text { R_fotohi@sbu.ac.ir; Fotohi.reza@gmail.com }}$

${ }^{1}$ Computer Engineering Department, University of Mohaghegh Ardabili, Ardabil, IRAN

${ }^{2}$ Center of Excellence in Future Networks, Iran University of Science and Technology, Tehran, IRAN

${ }^{3}$ Faculty of Computer Science and Engineering, Shahid Beheshti University, G. C. Evin, Tehran 1983969411, IRAN 


\section{Introduction}

Twitter is one the most popular microblogging sites, where users from everywhere can follow breaking news or posts about their interests. They can also post tweets with a limit of 140 characters about the topics they are attracted to. This is the reason we chose this site to extract information and knowledge about people's favorites in different geographical places. The dataset we used includes tweets of some users from mostly the United States of America, but it includes some other countries' tweets, like United Kingdom, Germany.

The major drawback of working with tweets is their shortness. Tweets with only 140 characters cannot be useful alone. To overcome this drawback, we use a dataset of tweets altogether, and instead of treating one tweet as one document [1], we treat a dataset of tweets with similar topics as one document. In this way, we also overcome the problem of not enough repetition of keywords which is mentioned in [2]. As long as treating a dataset of tweets as one document, TF-IDF can work properly, although the shortness of tweets was a problem to it [2]. Because keywords repeat more in these datasets TF-IDF values are more precise.

With the development of technology, news websites are taking the place of traditional news media, like newspapers. For example, www.bbc.com can be accessed by people from different spots on the earth, from Americas to Europe, Asia, or elsewhere. The arising issue now is how to cover all kinds of news from different places about all possible topics on news website homepages in a way to satisfy all kinds of people. Also, it must be considered that Internet users are interested in sites that show what they are looking for, or attracted to, sooner, and therefore it is the key to increase the popularity of websites.

In this paper, we represent a technique based on N-Grams and their TF-IDF values. Along with those values we use an effective coefficient to give higher $\mathrm{N}$-Grams more importance. This coefficient is chosen based on our training dataset, whereas the result separates relative topics from irrelative topics for a certain tweet. Then we use a test dataset and calculates 17 values, as we have 17 topic classes for every tweet. Based on the topic values, which are calculated in the previous step, we choose the relative topics. To do this, we calculate the distance between those topic values in order to select the relative ones. Our work was developed 3 times; 3-fold, 5-fold, and 10-fold development. The results showed the respective average accuracy percentage of 76.5163, 74.1117, and 68.4516.

\section{The main research contribution:}

- Obtaining the appropriate data set from tweets in the field of topic discovery

- Review of natural language processing methods and their use in message processing

- Review of machine learning methods for system training to learn subject discovery

- Analyze and obtain the percentage of interest of users in each region to each topic and find the most popular

The rest of the paper is organized as follows. Section 2 describes some preliminary, TF-IDF, one of the most practical keyword extractions, and N-Grams, a contiguous sequence of $n$ items from a given sequence of text or speech, Section 3 reviews related work. Section 4 goes through our data and method. Our experiment results are shown in section 5. Finally, the conclusions are presented in section 6. 


\section{Background}

In this section, basic concepts such as TF-IDF, and N-Grams are discussed in detail.

\subsection{TF-IDF}

In this method, words are given a weight based on their frequency in the document. In fact, this weighting system shows how important a word is to a document. This issue has many applications in data retrieval. The weight of a word increases with the number of repetitions in the text, but is controlled by the number of words in the text, because we know that if the length of the text is long, some words will naturally be repeated more than others, although very important in do not make sense [3]. This section is divided into two subsections: Term frequency and Inverse document frequency:

\section{A. Term frequency}

Suppose the number of times the word $T$ occurs in text $D$ is denoted by $\operatorname{TF}(T, D)$ and in the simplest case the initial number of iterations $T$ is denoted by $F(T, D)$. Therefore, Eq. (1) shows:

$$
T F(T, D)=F(T, D)
$$

The rest is defined as follows:

Boolean frequency: $T F(T, D)$ is equal to one if the word $T$ occurs in the text, otherwise it is equal to zero.

Logarithmic frequency scoring: Logarithmic frequency scoring is shown in Eq. (2).

$$
T F(T, D)=\log (f(T, D)+1)
$$

Augmented frequency: To avoid bias towards larger texts), i.e., due to the higher volume of the text than the other, the desired word may be repeated more, but this is not due to the greater frequency of the word in the larger text. $T$ divide by the initial frequency of each word that has the most frequency in the text, which is mostly used in the search engine to retrieve documents with the searched words. Therefore, Eq. (3) shows:

$$
T F(F, D)=\left(0.5+\frac{(0.5 * F(T, D))}{\operatorname{Max}\{F(W, D)\}: W \in D}\right)
$$

\section{B. Inverse document frequency}

It is a measure of the number of words that are very common in all texts and are usually repeated. The method of obtaining this criterion is that the logarithm divides the total number of texts by the number of texts including the common word. For example: Suppose there is 1000 texts in our entire database. If there is a special word in each of its $1000 \mathrm{~s}$, for example, the product of the logarithm of 
1000 is divided by 1000 , which means that it must be a common word and must have a coefficient of zero, but if the repetition has occurred in 500 texts, it is logarithm 2. The result is 1 . The greater the number of texts in which the word is repeated, the smaller the weight of IDF:

$$
I D F=\log \left(\frac{D}{(1+\{d \in D: T \in d\})}\right)
$$

With these explanations, the frequency of the image, the frequency of the document - is calculated as follows:

$$
T F-I D F(T, d, D)=T D(T, d) * I D F(T, D)
$$

\section{$2.2 \quad$ N-Grams}

In computational linguistics and probability, an N-Grams is an interconnected sequence of $\mathrm{n}$ elements from a given sequence of text or speech. Items can be called Phonemes, Syllables, Letters, Words or Base pairs, depending on the software. N-Grams are usually a text or speech collected from the Corpus. When items are words, N-Grams may also be called Shingles. An N-Grams, as large as one, is called a "Unigram"; Is the size of two "Bigrams" (or, to a lesser extent, a "Digram"; it is the size of three "Trigrams". "Four grams", "five grams", etc. are mentioned [4].

N-Grams models are currently widely used in Probability-Communication theory, Computational linguistics (for example, Statistical natural language processing Computational biology (for example, Biological sequence analysis), and Data compression. The two advantages of the N-Grams model are the algorithms they use (their simplicity and scalability. With a larger $n$, a model can store more content with a better understanding of the space of that content. People who are more interested in multi-word expressions may use this technique to pre-process strings to eliminate extra spaces. Many extra blank spaces are simply converted to a single space while the paragraph marks are removed.

$\mathrm{N}-$ Grams can also be used for word sequences or almost any other type of data. For example, they have been used to extract features to cluster a large collection of satellite images of the Earth and to determine what part of the Earth a particular image belongs to. It has also been very successful as the first step in searching for genetic sequences and identifying species from which short DNA sequences originate. N-Grams can be used in various fields of computer science, computational linguistics and applied mathematics [5]. This technique has been used in cases such as the following:

- Kernel design that allows machine learning algorithms, such as Support vector machine, to learn from disciplines.

- Find the correct spelling of words.

- Improved compression in Compression algorithms where a small area of data requires $\mathrm{n}$ grams longer. 
- Assess the probability of a sequence of words appearing in the text of a language in Pattern recognition, Speech recognition, OCR: Optical Character Recognition, ICR: Intelligent Character Recognition, Machine translation and similar programs.

- Improved retrieval in data retrieval systems to find similar "documents" with respect to a query document and a database of reference documents.

- Improve retrieval performance in genetic sequence analysis.

- Identify the language of a text or species from which a small DNA sequence is derived.

- Predict letters or words randomly in order to create text.

- Cryptanalysis.

\section{Related work}

Text classification and filtering are important aspects of this research. In this section, we will review the classification of short texts, as it is a very important topic about the classification of microblogging messages. In the second part, we will have an overview of Twitter research. Finally, we review the big data, because due to the time complexity of this research, from our point of view, it is in the big data section. In each section, first a brief explanation of the relevant topic is provided, then we refer to the relevant papers.

\section{A. Classify and filter}

With the daily increase of news sites, receiving emails with the theme of daily news or sometimes moment-by-moment news, can become Information overload. Because different news sites try to attract users to increase their popularity, but in this case, users get much more news by receiving too much news, which usually includes their favorite and disliked news. They can search for their favorite topic, and in this case, the subscribers of news sites will be reduced. On the other hand, users who visit news sites are more inclined to those who often present their favorite topics on their homepage. This raises the need for an information filtering system based on users' interests.

A review paper on text filtering is provided in [6]. We have two types of text filtering systems: Content-based system and Social-filtering system. The first type is how to filter based on information extracted from texts. The second type is based on notes taken by previous readers about the text. According to the framework presented in this research, the relevant system is text-based. Text filtering can be considered as a program in which documents are divided into two classes: Relevant class and Irrelevant class.

Reference [7] provides an overview of Machine learning for automated classification and explains how classrooms are constructed and evaluated for this classification model, text classification. Restrictions on the size of texts in microblogging services result in very little data in the field of classification. This issue exists in other areas such as Web search snippet, Web search snippet and Chat services.

Different theories have been proposed to solve this problem, such as using Wikipedia as an external source [8] and [9], or using the results of network search engines [10]. 
Another solution [11] uses Topic models. In this theory, a thematic model is constructed using a Latent Dritchlet [12] Allocation on a general corpus, and then this thematic model is used to classify short texts.

Another solution, which is a method for measuring the syntactic similarity of texts using knowledge-based and corpus-based measurements, is presented in [13].

\section{B. Twitter}

Microblogging is an emerging form of communication and has become very popular in recent years. Micro-blogging services allow users to publish short messages. These messages are sent to their Followers immediately. In this study, our focus is on Twitter, the most popular microblogging service with more than 144 million users by 2414. In recent years, microblogging services and social networks have become very popular fields for research. Twitter is the most popular microblogging service, but the main reason it is popular with researchers is its access to data. Unlike most social networks, the messages and comments of the users of this site are displayed to the public and can be retrieved in real time using the Twitter application interface. Relationships between members in this network can also be restored.

It has been studied why users use Twitter and how communication is formed. In this paper, the topic is that daily chatter, conversations, sharing information or URL, and news reporting are the main goals of users.

A study has been conducted on the uses of Twitter, exploring its benefits and its impact on informal or verbal communication [15]. Most research on Twitter has focused on analyzing, summarizing, and exploring real-time data.

References [16] and [17] discuss the collection and evaluation of real-time related negotiations during that event.

References [18] and [19] describe systems that collect and index large amounts of text data, such as new messages on blogs, Twitter messages, and news. These systems also allow online analysis of this type of data.

A system for discovering news messages on Twitter and clustering these messages based on their position in [24] is provided. As a real-time system, Twitter is a good source of new information or content. So, its real time stream can be used to find new pages. Search engines often use this method to find new pages to show them in their results.

References [21] and [22] use microblogging to detect new network addresses and improve search engine rankings.

\section{Big data}

When we talk about big data, we can only mistakenly think that we mean only very large volumes of data. Bulk data is not a new issue. Wal-Mart's corporate data warehouse was about 300 terabytes in 2003 and 480 terabytes in 2004. These tanks were really bulky at the time, in contrast to the 
techniques available at the time. So, it's not just the volume of data that counts, but the core meaning of "big data" is the performance of the equipment compared to what it needs to do, so what kind of data needs to be analyzed. Also, all previous methods worked on structured data, but big data often works on unstructured data.

Several papers have been published on the potential of big data, emphasizing the possibility of a revolution in science and society as a whole. Community white paper Written by several American management researchers states that a well-directed, well-directed investment in big data can not only lead to great advances in science, but also establish a generation. Next will be advances in science, medicine, and commerce [23].

Alex Pentland, director of the Human Dynamics Laboratory and one of the pioneers who has used vast data in the social sciences, claims that this data can be a great tool for Reinvent society and can improve its processing [24].

While the rest of the researchers talk about big data, they mean relationships on social media, Alex Pentland focuses on the location of data from mobile devices, payment data from credit cards, and so on. Be. He sees this data as data to describe the actual behavior of humans, not their choices to relate to others. In his view, big data is ascending about the actual behaviors of individuals [24] and the communication of individuals.

This potential for community restructuring has also been recognized by the mainstream media and has been widely discussed. The New York Times, for example, published "The Age of Big Data" [25] and published books on how big data transforms our way of life, work, and thinking [26].

Various social media sites are often used as data sources in social media research and social behaviors. However, these data do not represent the entire human population. It may have a specific orientation to a country, an age group, or people who deal with technology in general. In addition, researchers may not even have access to the entire population on social media [27].

The standard Twitter application interface, for example, does not retrieve all tweets, but only selects and retrieves some of them, and the search application interface retrieves only recent tweets [28].

\section{Proposed approach}

In this section, the proposed idea is explained in detail in three subsections: classification and filtering, possible changes in existing techniques, and big data.

\subsection{Classification and Filtering}

Classification and filtering are two very similar concepts. For example, we can say that an expression can belong to a class "A" or not. It can be said that the phrase is filtered if it does not belong, or it can be said that the phrase is being classified. Now consider a news system whose news topics are limited to 17 topics. People from all over the world have the ability to connect to this system and view its news. Of course, there can be several headlines a day about some news 
items. Now including all these headlines on the main page of the site is one issue, another issue is how this news is arranged. Because news "A" can be considered important news for a country like Iran but not important for another country like Sweden. So, the news layout should be changed according to the user's geographical location or even some of this news should be filtered.

On the other hand, such news systems sometimes send daily news to their subscribers. If this news is not in line with the users' interest, the number of subscribers will decrease over time. Therefore, the need for a News Filtering System, or it can also be called a news suggestion system, is felt to filter the submitted news according to the relevant user or change the arrangement of the news according to the priority of users' interests. To this end, this article provides a way to find the topics of interest to the residents of those areas based on the tweets of users in different geographical locations, and to use these results to filter the news, either on the main page of the site or in sending E -mail, used. These results can also be used to change the order of news based on the priority of users' interests. Thus, we first need to find topics related to a tweet, because these topics speak to the interest of their authors. Then, according to the geographical location of the users and the topics found, the topics of interest of the majority of the residents of those areas, who are related to online news media, are found.

The proposed classification has two phases: Feature extraction phase, which includes 5 stages, and Topic discovery phase, which includes 6 stages.

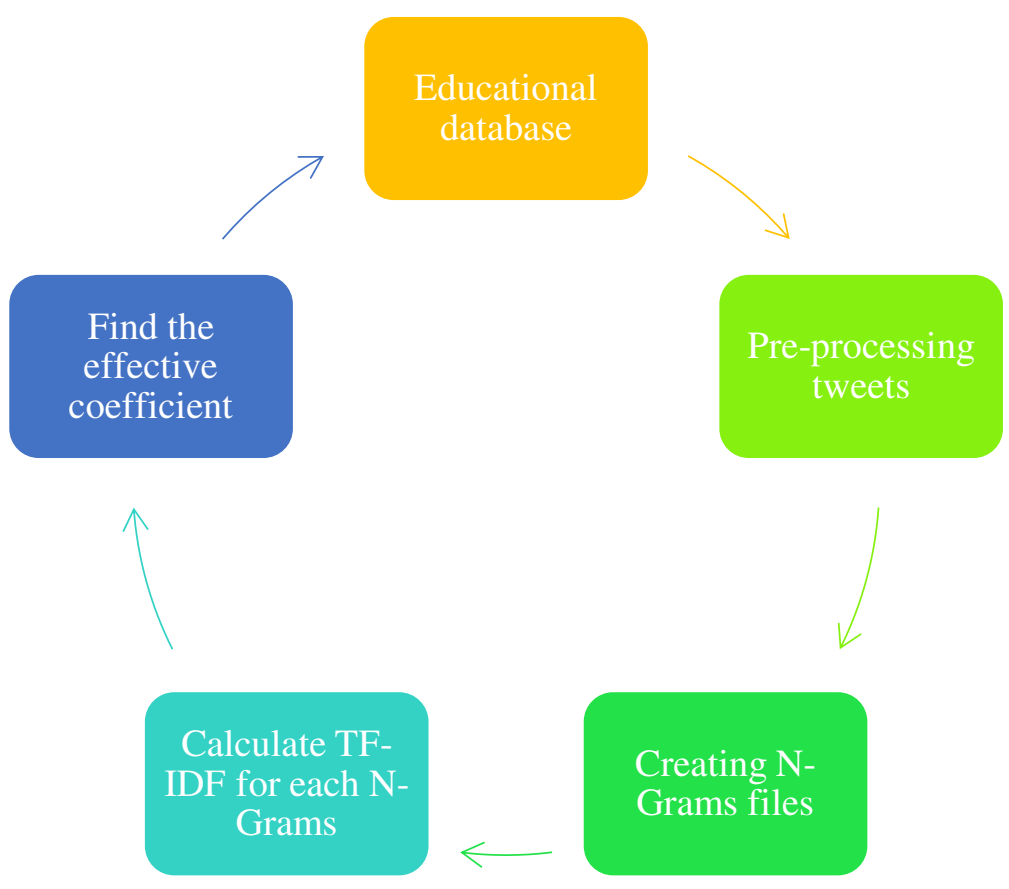

Fig. 1 The first phase of the proposed model, finding the effective coefficient for better weighting of $\mathrm{N}$ Grams.

\subsubsection{Feature's extraction phase}

This phase is in fact the educational phase of this article and consists of 5 stages: 
Educational data group: In the first step, a series of tweets are randomly selected. At this stage, each tweet should be accompanied by relevant topics. These topics can be found and added manually or, as done in this article, the topics can be identified by the Thomson Reuters Institute and added to this data set. These tweets are used to find N-Grams related to each topic and then weighted. The N-Grams found along with their weight are used as features that are calculated using the Topic discovery phase.

Pre-processing tweets: We need a series of edits to extract the N-Grams related to a topic. First of all, we have to remove Stop words. Stop words are words that are common to all topics. At this stage, we need a natural language processing tool to do this. Using this tool, we first delete Stop words throughout the data set, then use the Regular expression package, which is common in the Python language, to filter the characters.

Making N-Grams Vectors: For each topic, we create separate vectors of all the unique N-Grams that we found in the topic data group in step A. In this article, we consider the value of $n$ to be between 1 and 30. As a result, depending on the length of the tweet, up to 30 vectors can be found for it. In other research, they have created a vector with the whole N-Grams community and used it to find similarities between a tweet and vectors of different topics. The reason for not using this method in this article is to save memory and search time.

Calculate TF-IDF for each N-Grams: At this point, the goal is to find a weight for each attribute, $\mathrm{N}$-Grams. One of the simplest and most widely used methods is to use TF-IDF. In last research, it is mentioned that this method is very useful in text documents, such as news articles, because in these texts we want to find phrases that are repeated frequently in these documents, but are not common in other existing documents. To be. Of course, there is a negative point in that article that this method is not suitable for working with Twitter, because the tweets are very short, and therefore most phrases and words, including keywords, are repeated only once. be. In this article, we do not consider a tweet as a document in the training phase, but consider the Corpus of tweets with the same topics as a document. With this approach, we achieved satisfactory results.

Find Effective Coefficient: This stage is the most important stage of this phase. In the course of this research, we conclude that if the significance of $\mathrm{N}$-Grams increases exponentially with increasing $\mathrm{n}$, in the subject discovery phase, relevant and irrelevant topics are broken down into two separate groups. The effective power coefficients are $\mathrm{n}$ in $\mathrm{N}$-Grams. In implementing this research, the best result was obtained with $n^{6}$.

\subsubsection{Subject Discovery Phase}

This phase is the experimental phase and has 6 stages: 
Educational data group: The first step in this phase is to select the remaining tweets from the total data set so that the training tweets are deleted. This new data set is used to test and execute algorithms on them.

Pre-processing tweets: In this step, we first remove the word Stop, words that are common to all topics. To do this, we need natural language processing tools. Using the Stop word list of this tool, we first filter them across the data group, then use the Regular expression package to filter the symbols.

Find N-Grams: All N-Grams are found in one tweet. The value of $\mathrm{n}$ is between 1 and the total number of words in the tweet. Of course, it should be noted that the maximum value that $\mathrm{n}$ can have is 30. These N-Grams are used to measure the similarity between relevant tweets and different topics.

Aggregate Effective Coefficient * TF-IDF values for a tweet: In this step we use the N-Grams found in the previous step and find the equivalent vectors, if the equivalent vector is available. To do this, the search is performed only on vectors that have the same value $n$. If these vectors were present, we would find the result of the sum of their TF-IDF multiplications, calculated in the fourth stage of phase 1 , with the effective coefficient, calculated in the last stage of phase 1 based on the value of n. Eq. (6) shows this calculation.

$$
\text { result }_{\text {tweet }_{i}}=\sum_{k=1}^{\max (n)-(n-1)} T F-I D F_{k} * E C_{n}
$$

Sort the results of all topics: The results in this step are the sums obtained in the previous step. For this purpose, we arrange these results in descending order. The largest value indicates that the relevant topic certainly belongs to the relevant tweet. In the next step, other relevant topics will be found.

Calculate the distance of the results: As mentioned before, the first issue is the biggest result. To get the other topics, we move forward in the sorted list and find the distance of each member to the first value, which is labeled as the first relevant topic. If the distance obtained is greater than 99, it indicates that the subject is one hundred percent irrelevant. With the first interval of 99, the algorithm does not continue and stops. Eq. (7) shows this calculation.

$$
\text { topics }_{\text {tweet }_{i}}=\left\{S R_{1}, S R_{t} \mid S R_{1}-S R_{t} \leq 99\right\}
$$




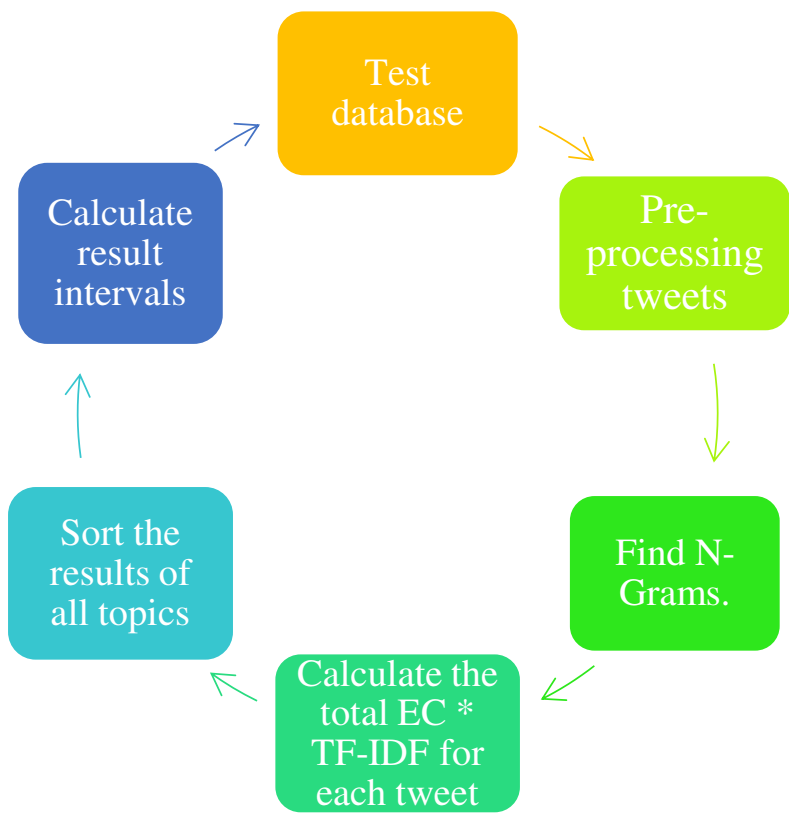

Fig. 2 The second phase of the proposed model is to calculate the distance between the results of the similarity of a tweet and different topics.

\subsection{Possible changes in existing techniques}

\subsubsection{How to use N-Grams}

One of the most important techniques used in word processing is the use of N-Grams. N-Grams are a sequence of words that are separated by $n$ sentences. In this study, the value of $n$ is between 1 and the maximum number of words in a tweet, although this value is not defined more than 30 . It should be noted that word order does not matter here, so N-Grams are arranged in alphabetical order to avoid repetition due to word rotation. Each N-Grams is stored in a separate list with N-Grams of the same size to avoid additional comparison time when searching. N-Grams can be used as a Feature for texts. This means that by using the common N-Grams between a tweet and the N-Grams in a topic, you can determine the similarity of that tweet to that topic. Also, because the higher the number of words in N-Grams, the more accurate they contribute to this problem. We change the effect of these expressions according to the value of $n$ with an Effective Coefficient. That is, the higher the $\mathrm{n}$, the greater the effect. In implementing this research, we obtained the optimal answer when this coefficient of impact increases exponentially and is calculated with $n^{6}$.

\subsubsection{TF-IDF: Term Frequency-Inverse Document Frequency}

TF-IDF is one of the easiest ways to find keywords and weight words and phrases in text processing. In this method, the weight of a word or phrase is based on the amount of repetition in a document, while the amount of repetition in other documents are not ineffective. This method has been criticized in many articles and researches for its use in the field of Twitter, because the tweets are very short and the keywords are often repeated once in a while. To address this shortcoming, what has been done is to use the body of tweets as a document, rather than a tweet as a document. Also, as mentioned in the previous section, the weights attributed to N-Grams change with respect 
to the value of $n$, which means that by increasing the $n$ frequency of the inverse expression, the frequency of the document increases exponentially, with an impact factor.

\subsubsection{KNN: K-Nearest Neighbor}

The nearest neighbor $\mathrm{K}$ algorithm is used in this paper in two steps. To classify a tweet, we need to give the tweet weight to each topic. To do this, the $\mathrm{k}$ algorithm finds the same neighbor in each Topic class, these neighbors are the same N-Grams in each subject, and we add them TF-IDF. This is the sum of the weight of that tweet relative to that topic. This is the first step in using this algorithm. The next step is to find topics related to a tweet. This means that the weights given in the previous step of the relevant subject classes, the nearest neighbor $\mathrm{K}$ algorithm, must be found. The nearest neighbor $\mathrm{K}$ algorithm used in this study has little change. This change is applied only to the value of $\mathrm{K}$, so that in the standard mode of the algorithm, this value is a fixed value specified by the user or programmer. But here in both steps the value of the $\mathrm{K}$ algorithm varies and this value changes as the tweet changes. This value is not set manually, but the program finds it dynamically and intelligently. For example, the phrase "food prices have gone up with rising inflation" could be about Business, Finance and Politics, i.e., $\mathrm{K}$ is equal to 2 , but the phrase "tourists without visas are at risk of terrorism and the exchange rate falls in the country" Can be in the case of business and finance, Disaster and Accident, Law and Crime and politics, i.e., $\mathrm{K}$ is 4 . So, we see that the existence of a constant value as $\mathrm{K}$ is not reasonable and we needed this change in this algorithm. How to find the value of $\mathrm{K}$ in the algorithm is that in the first step we used to determine the number of N-Grams similar to this value, and in the second step of using this algorithm, the distance between the topic weights of each the tweets, which were described in the second phase of the proposed algorithm of this research and in step 6, specify this value.

\subsection{Big data}

As mentioned earlier, there are three main reasons for using big data: Volume Information, Velocity and Variety Data. The reason for this research is in the field of big data, low processing speed and searches. The data group processing speed used in this study increased dramatically when the project was transferred from Python to PySpark. Also, due to the use of more RAM, the search time was greatly reduced. Figure 3 shows the architecture for using large data using the PySpark tool.

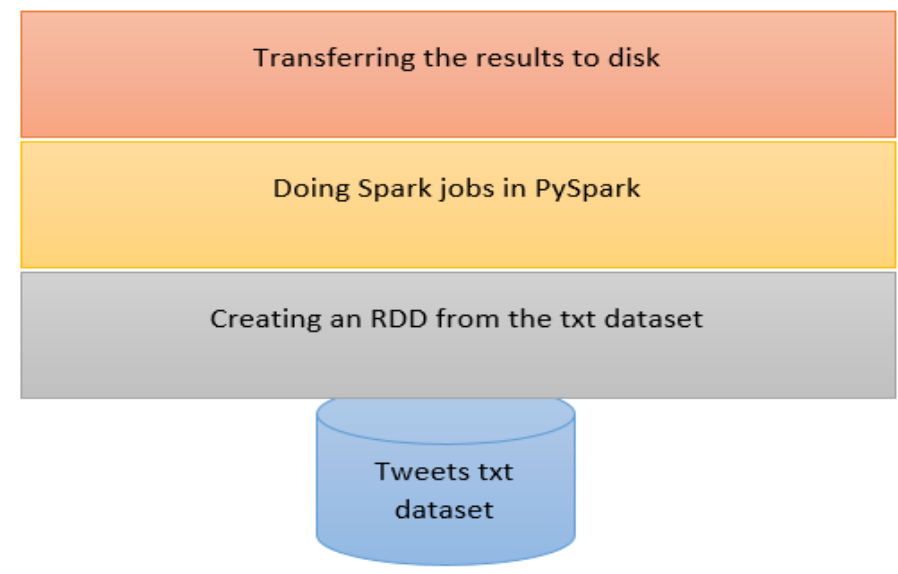

Fig. 3 Proposed system architecture in the field of big data. 


\section{Performance evaluation}

\subsection{Implementation features}

In this article, we used natural language processing tools and PySpark. Natural language processing tools are used to pre-process tweets in this article. For example, to delete symbols or delete Stop words. Also, because the method presented in this article has nothing to do with the order of the words, this tool has also been used to sort the words of a phrase in alphabetical order, because if you do not sort the phrases into the same phrases with different permutations from, we encounter the same words.

This paper was first implemented in PyCharm in Python. But due to the very high time complexity, which is due to the large amount of data, it was transferred to Spark and reimplemented in PySpark in Python. The advantage of using Spark is faster speed in required searches. Because it brings more data to RAM and speeds up these searches. In this part of the article, the results are obtained in the form of graphs in MATLAB software. The implementation of the idea presented in this article is in three ways. In the first case, we consider $10 \%$ of the total data as educational data sets, in the second case, $20 \%$, and in the third case, approximately $33 \%$ of them are educational data. Each of these implementations has been compared with each other in terms of the accuracy of the results, and each implementation within itself has been compared in terms of the accuracy of the results by changing the training dataset. Also, the percentage of topics of interest to users in different geographical areas is shown on the chart.

\subsection{Implementation data}

The data used in this research is a data set that was introduced and made available to the public by [3]. According to the same article, the dataset includes tweets that have been themed by the Thomson Reuters Center and used for research. It should be noted that only the values of tweets, subject, and geographical location of users in the relevant data set are used. Also, tweets without geo-location names have been filtered because the goal was to find out what users might be interested in. The number of topics covered in this dataset by the Thomson Reuters Center is 17, which are as follows:

Business_Finance, Disaster_Accident, Education, ntertainment_Culture, Environment, Health_Medical_Pharma, Hospitality_Recreation, Human Interest, Labor, Law_Crime, Politics, Religion_Belief, Social Issues, Sports, Technology_Internet, War_Conflict, Weather.

Finally, diagrams are shown to show the percentage of interest of users in different geographical locations to different topics.

\subsection{Implementation results}

The presented diagrams are based on the accuracy of the algorithm, which is measured in all 3 types of implementations. This accuracy has been measured in subject-based, instructional-based, and implementation-based modes. This accuracy means the ratio of the number of tweets whose topics 
are correctly guessed to the total number of tweets on a particular topic [29]. Eq. (8) shows this calculation.

$$
\text { Accuracy }=\# \text { correct results } / \# \text { all tweets }
$$

Finally, diagrams are shown to show the percentage of interest of users in different geographical locations to different topics.

\subsection{Average accuracy charts}

Figure 4 shows the average accuracy of the algorithm in three modes of 3, 5 and 10 parts (3-fold, 5fold, 10-fold). In the first case, the educational data set is approximately $33 \%$ of the total data set with an average accuracy of $76.5163 \%$. In the second case, $20 \%$, with an average accuracy of $74.1117 \%$, and in the third case, $10 \%$, with an average accuracy of 68.4516 , is the total data set. In the opinion of this research group, the best implementation has been the 5-fold mode, due to its better accuracy than the 10-fold mode and the smaller training data set than the 3 -fold mode.

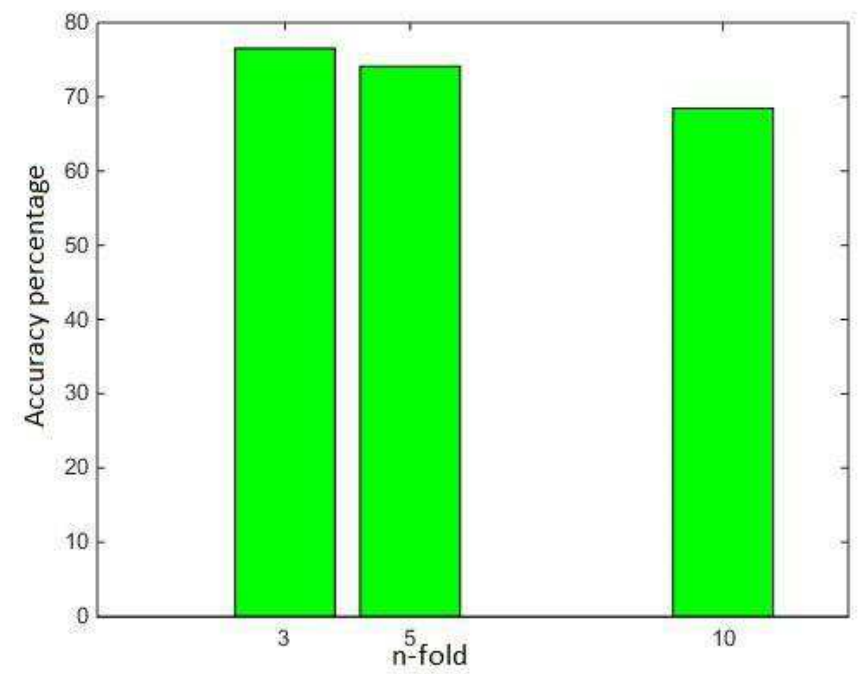

Fig. 4 The average accuracy of the algorithm in three modes of 3, 5 and 10 parts.

Figures 5, 6 and 7 show the average accuracy of the algorithm in different implementations according to different training data sets. In Figure 5, we see that when the first part of the data set is considered as an educational data set, the final results are more accurate and better. This shows that with this scatter of words and phrases we can achieve more normal frequencies. The distance between the best and the second-best results is 3.4477. In Figure 6, section 5m, with a slight difference from the second best, 1.9657, is the best section for the training dataset, and in the last figure, Figure 7, as can be seen, forms the sixth section of the best training dataset. In all of these graphs, the result bars are curved together, with the initial and end bars being the longest and the middle bars the shortest. This indicates that in this dataset the words and phrases are scattered more 
normally at the beginning and end than in the middle of the whole data set. As a result, more normal values are obtained for the frequency of the inverse expression of the document, in the case where the training dataset is at the beginning or end of the whole dataset.

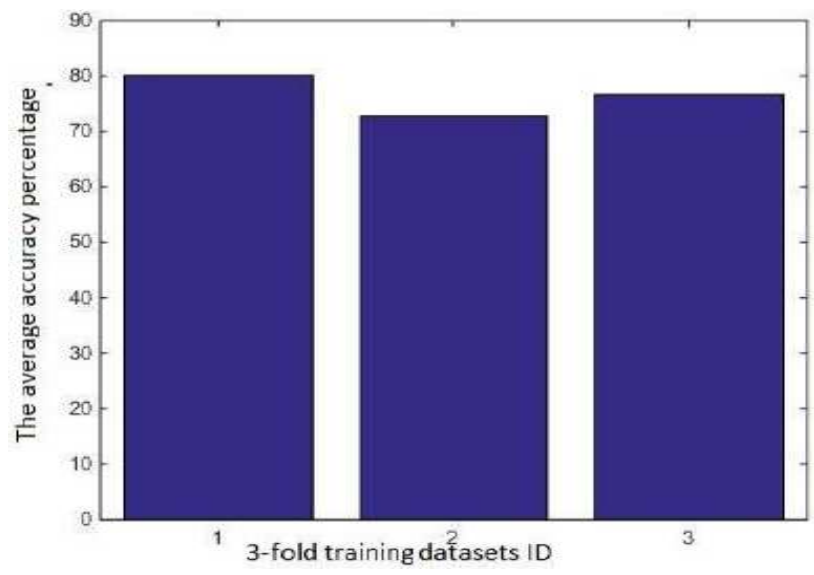

Fig. 5 Average accuracy in 3-part mode.

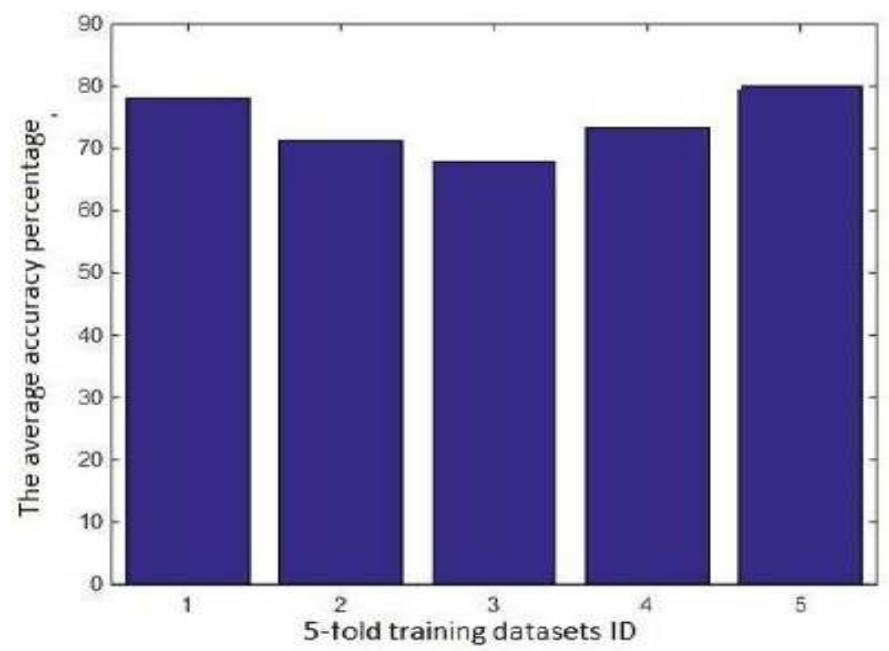

Fig. 6 Average accuracy in 5-part mode.

Figure 8 shows the average accuracy of the results with respect to different subjects. In the corresponding diagram, we see that accuracy is directly related to the size of the training data set. The larger the training dataset, the greater the accuracy. But one point in this diagram shows that it all depends on how the words and phrases are scattered in the data set. As we can see in the diagram, in the last issue, although the size of the training dataset has increased from $20 \%$ to approximately $33 \%$, the accuracy has decreased. This shows that the way the words are scattered, a higher percentage of words are words and phrases that are not related to the relevant topic, in the case of 3 parts, the frequency of TF-IDF is more inappropriate, and thus the accuracy of the results the result is reduced.

Figures 9, 10, and 11 show the average accuracy of subject-based results in 3-fold, 5-fold, and 10 -fold implementation modes. The data displayed in these diagrams show how words and phrases are distributed throughout this dataset. The best word scatter in these graphs is when the result 
points in a particular topic are on the vector of an instructional data set as close to the mean point of that topic as possible on the mean vector. The greater the distance between the subject vectors as well as between them and the mean vector, the greater the indication that the word scatter is not the same in different parts of the data set, and therefore the frequency of the inverse expression is the frequency of the document in a word. Different values of the data set, which are considered as educational data sets, are calculated different values. To see more closely the scatter of words and phrases in this dataset, their standard deviation is calculated and displayed in a table. These results are shown in Table 1. Based on the results of standard deviation, the best dispersion of words and phrases in the subject of crime-law.

Table. 1 Standard deviation of the results of each issue in three implementation modes: 3-fold, 5-fold, and 10-fold.

\begin{tabular}{|c|c|c|c|}
\hline $\mathbf{N}$ & \multirow{2}{*}{3} & \multirow[t]{2}{*}{5} & \multirow{2}{*}{10} \\
\hline Topic & & & \\
\hline $\mathbf{0}$ & 1.3788 & 4.1639 & 7.8792 \\
\hline 1 & 8.0569 & 3.4723 & 5.3612 \\
\hline 2 & 4.6733 & 6.5595 & 7.9003 \\
\hline 3 & 8.0530 & 7.7305 & 8.9134 \\
\hline 4 & 2.3058 & 5.5952 & 7.2203 \\
\hline 5 & 6.9168 & 8.1812 & 9.3542 \\
\hline 6 & 6.6508 & 6.2147 & 7.2877 \\
\hline 7 & 7.4154 & 7.7691 & 8.8573 \\
\hline 8 & 6.0671 & 5.2655 & 7.8250 \\
\hline 9 & 2.0660 & 2.9574 & 3.6604 \\
\hline 10 & 5.9351 & 5.7653 & 7.3714 \\
\hline 11 & 7.3262 & 7.4558 & 7.2431 \\
\hline 12 & 5.9839 & 6.3729 & 6.7886 \\
\hline 13 & 2.5573 & 4.3996 & 7.8310 \\
\hline 14 & 5.3834 & 7.7240 & 9.3704 \\
\hline 15 & 6.0807 & 3.5500 & 5.7081 \\
\hline 16 & 5.6024 & 5.8374 & 11.0277 \\
\hline
\end{tabular}




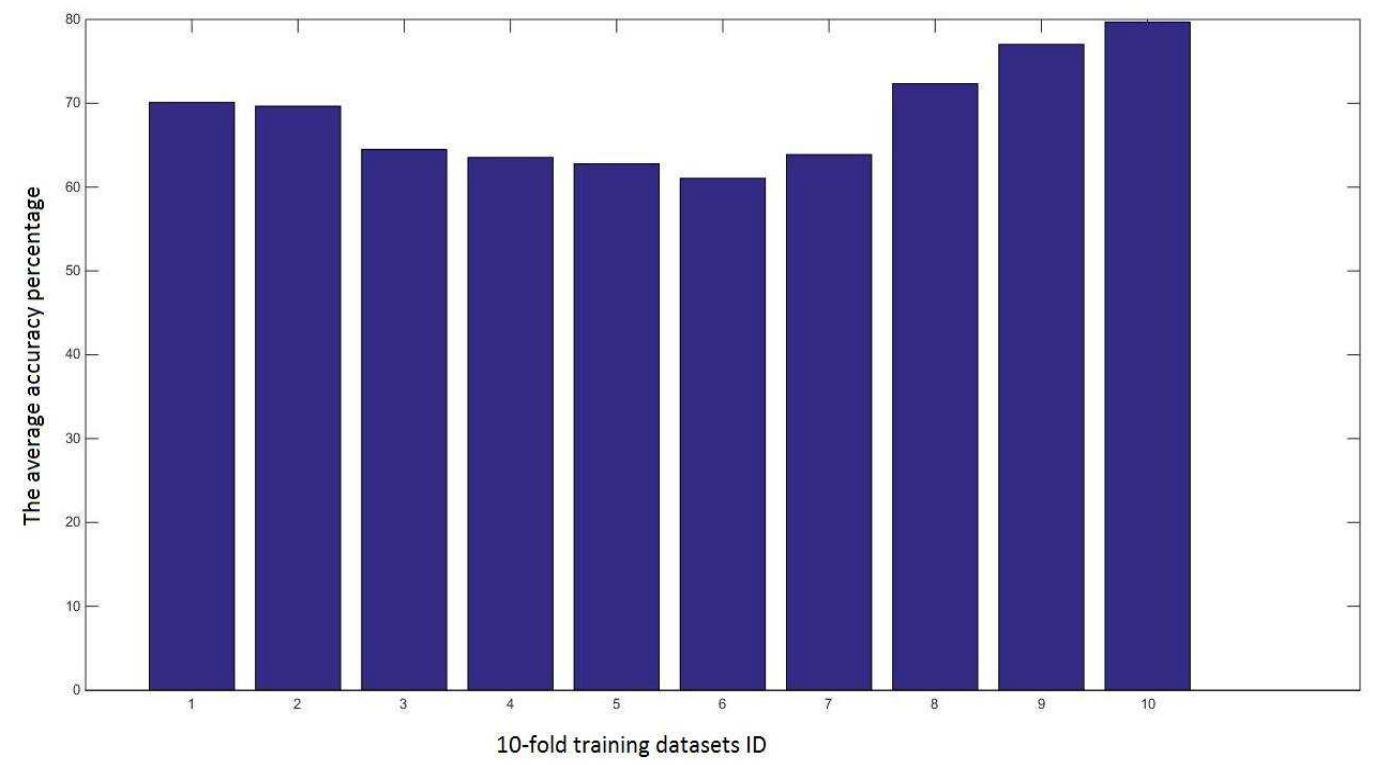

Fig. 7 Average accuracy in 10-part mode.

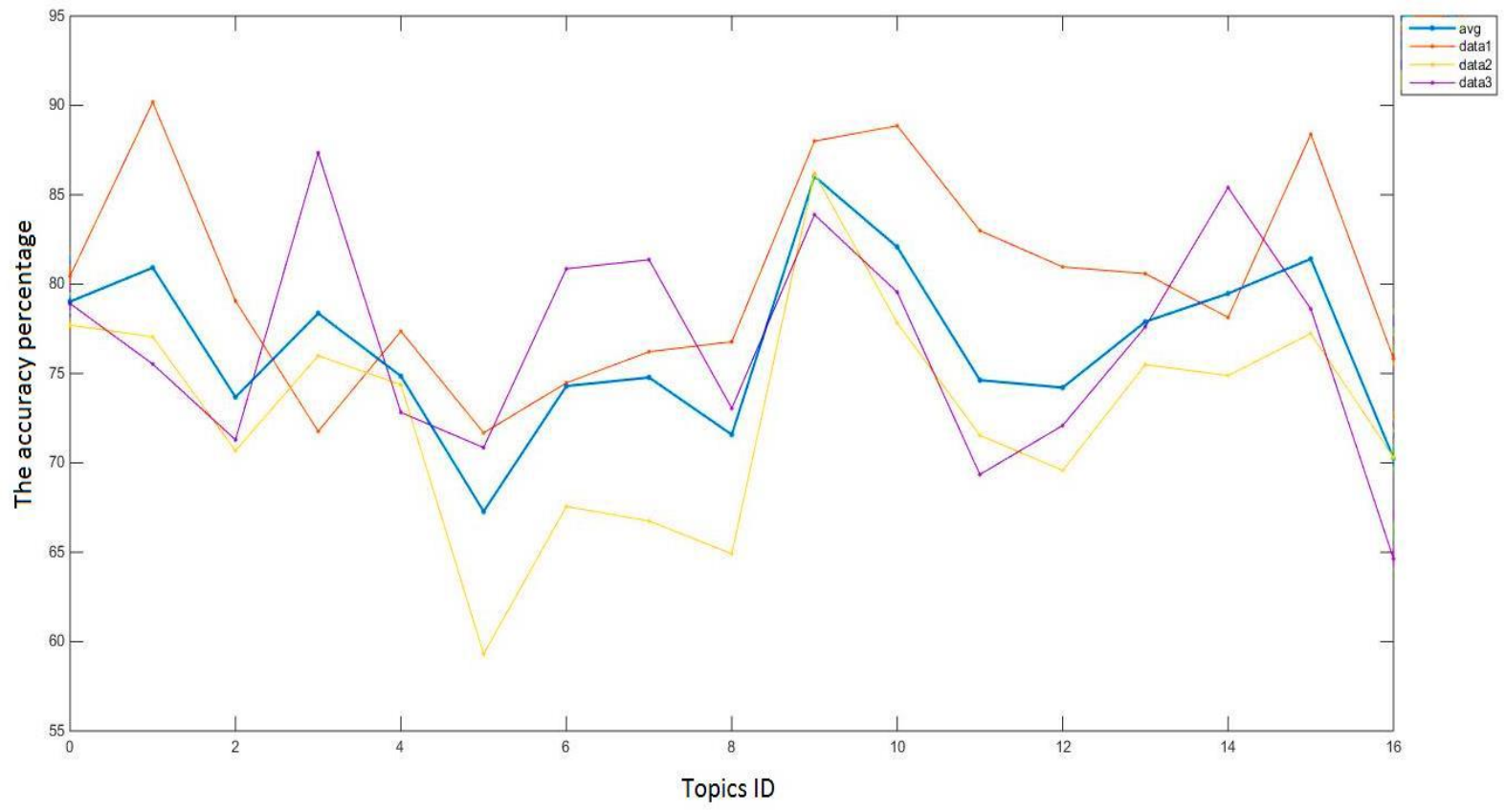

Fig. 8 Average accuracy with respect to different subjects for all three 3-part mode, 5-part mode, and 10-part mode. 


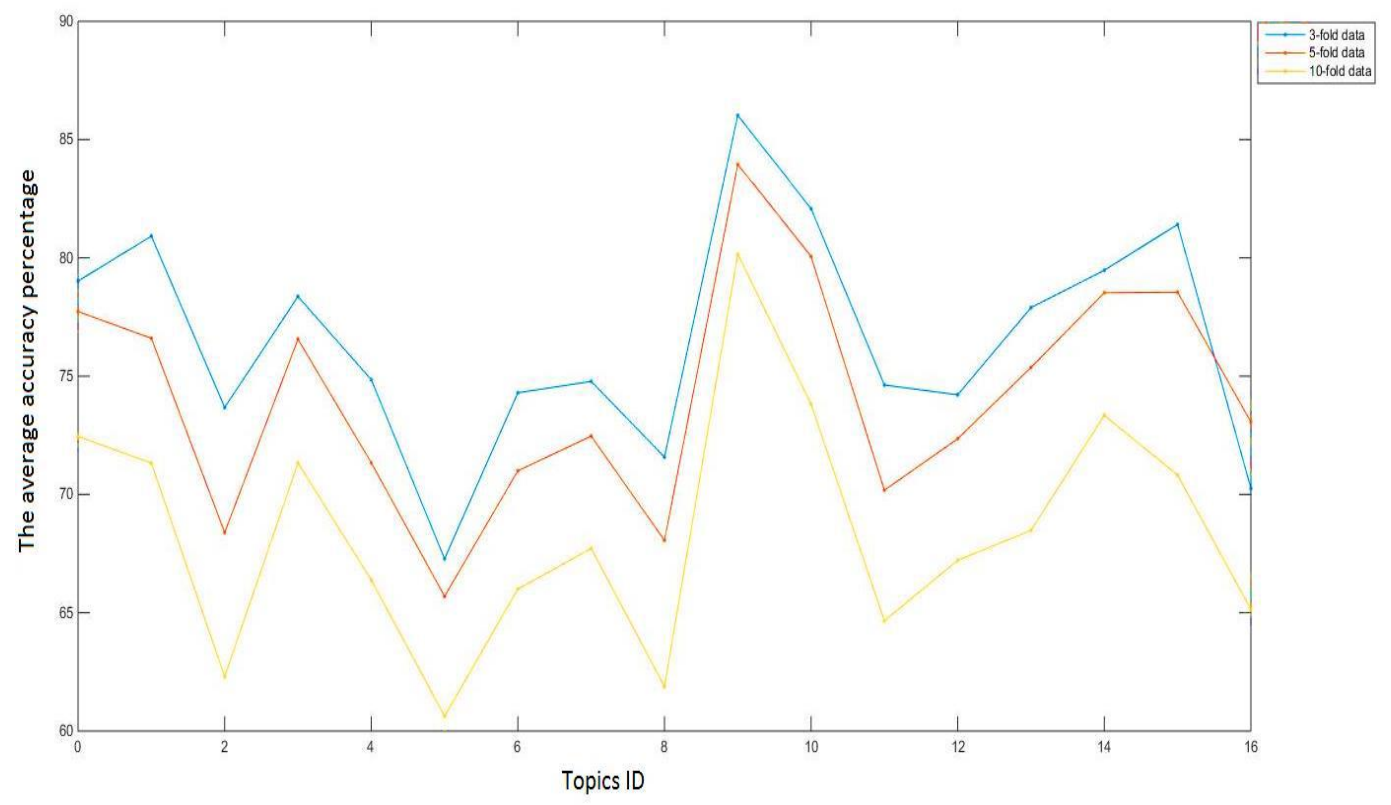

Fig. 9 Average accuracy according to different topics for 3-part mode. This diagram shows how words and phrases are scattered.

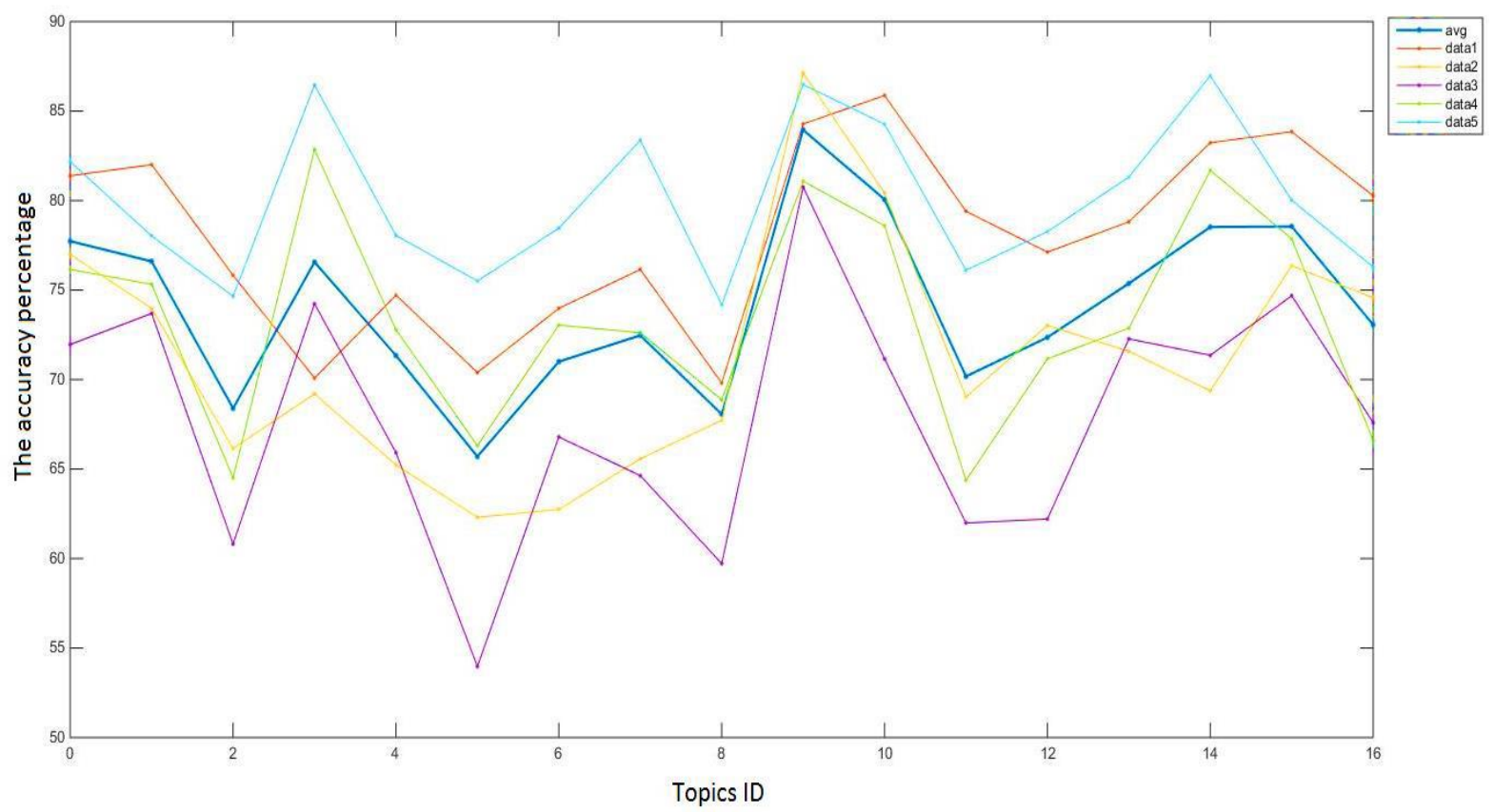

Fig. 10 Average accuracy according to different topics for 5-part mode. This diagram shows how words and phrases are scattered. 


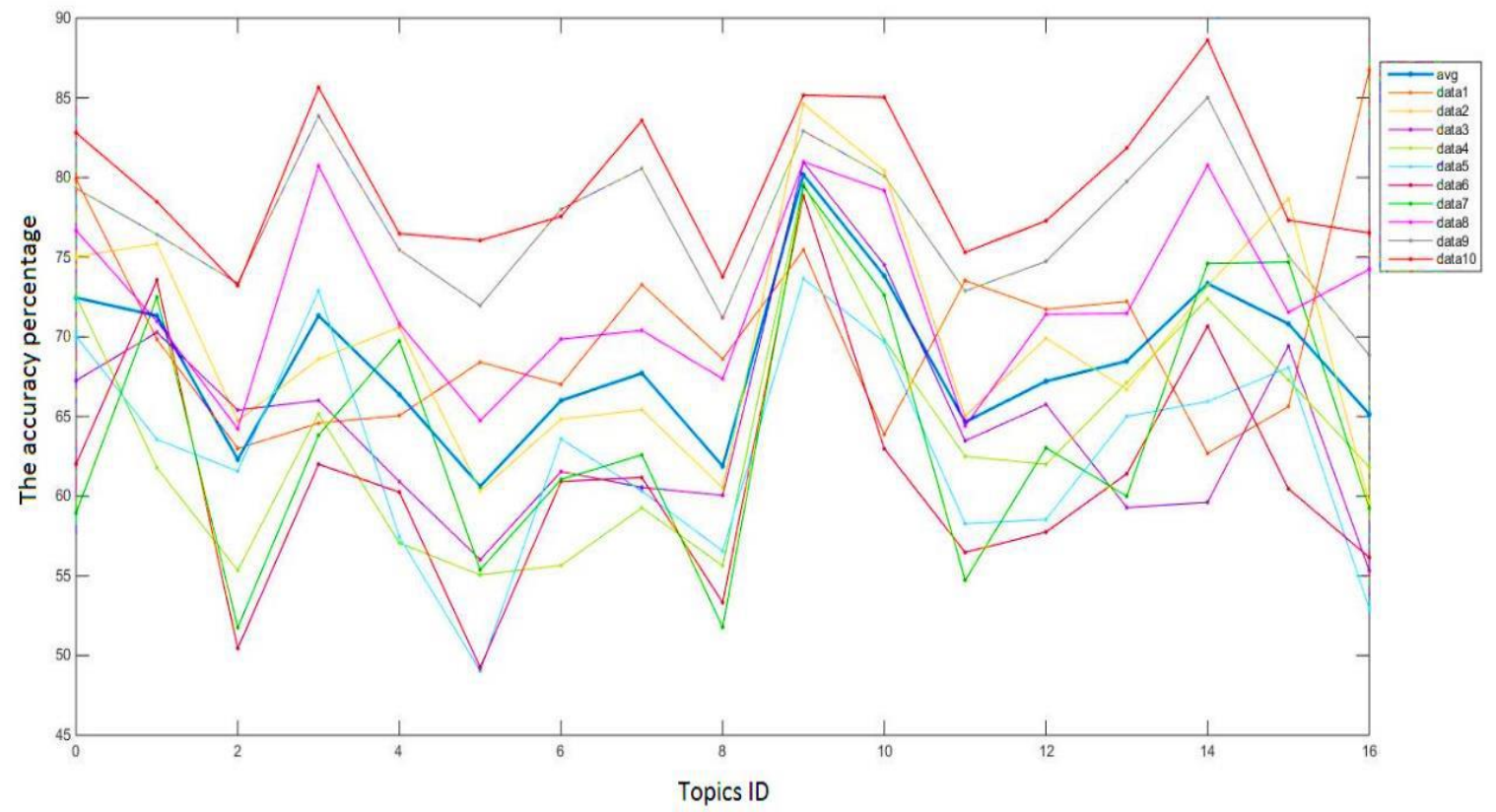

Fig. 11 Average accuracy according to different topics for 10-part mode. This diagram shows how words and phrases are scattered.

\subsection{User interest percentage charts}

The purpose of this study was to design a news suggestion system using the discovery of topics of interest to Twitter users and based on their geographical location. For this purpose, tweets related to several countries have been used for example. The final results for each country are shown separately in Figures 12 to 28. Finally, Figure 29 presents the final results in three dimensions with the axes of topics, countries, and percentage of interest. For example, it can be seen that Twitter users in Italy have talked more about the number one topic, business-finance, or the first topic of interest for American users is entertainment-culture. Tables 2 and 3 also show the identification numbers of countries and topics with the equivalent of their names.

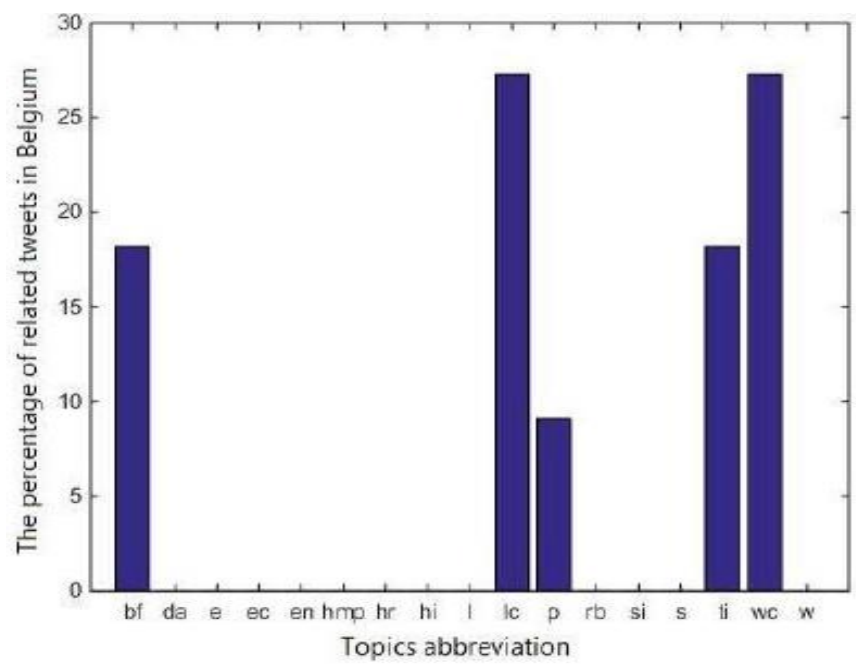

Fig. 12 Percentage of Belgian users interested in various topics. 


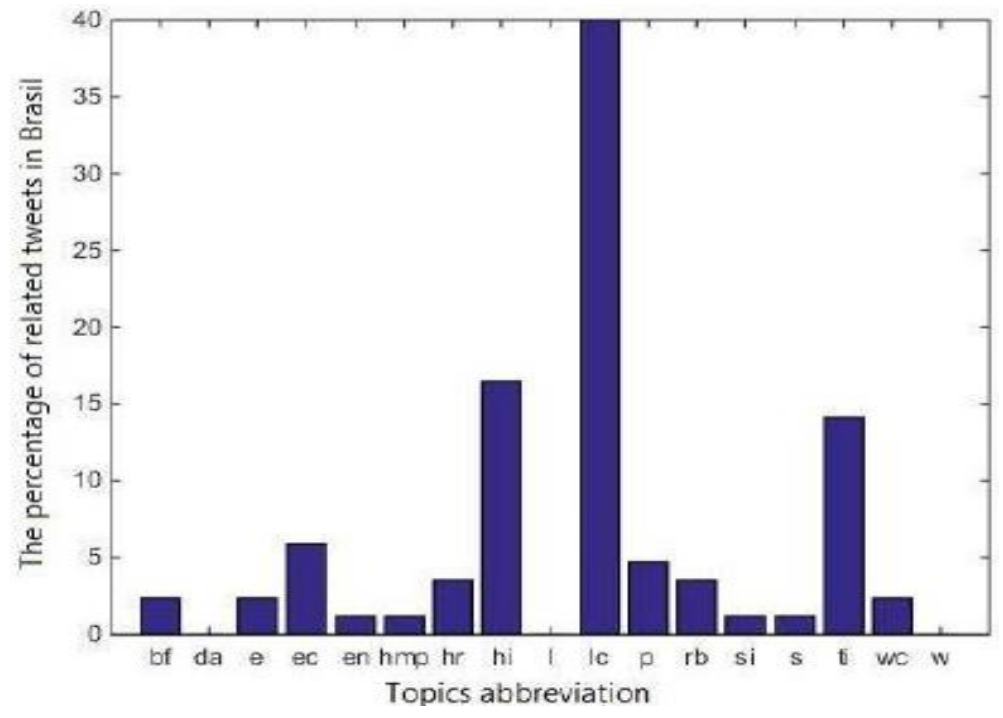

Fig. 13 Percentage of Brazilian users' interest in various topics.

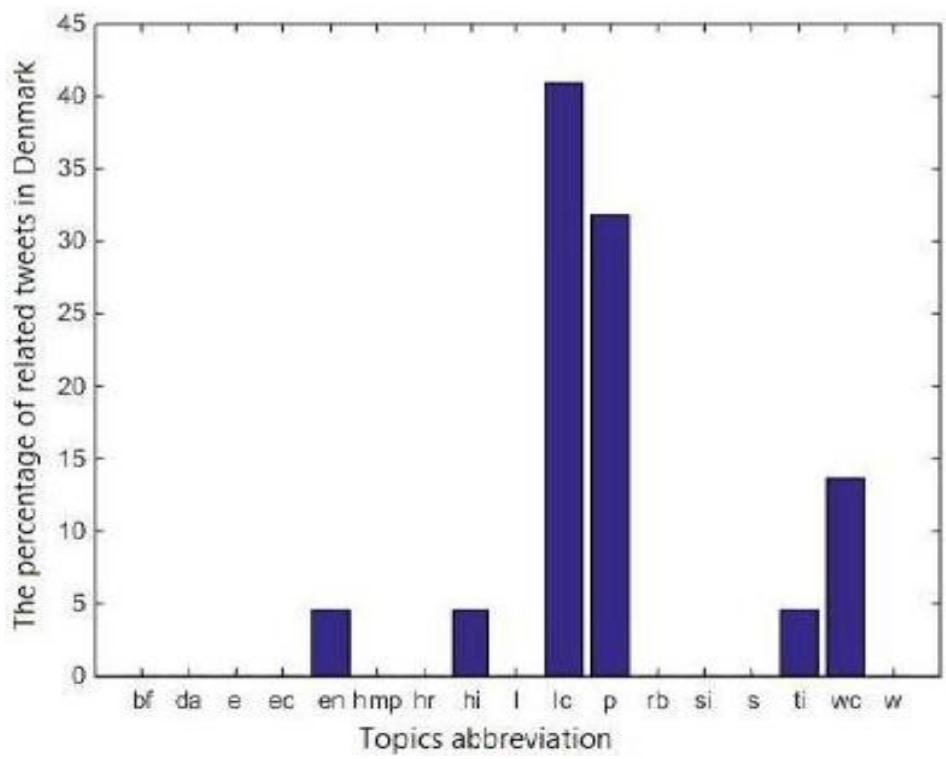

Fig. 14 Percentage of Danish users' interest in various topics. 


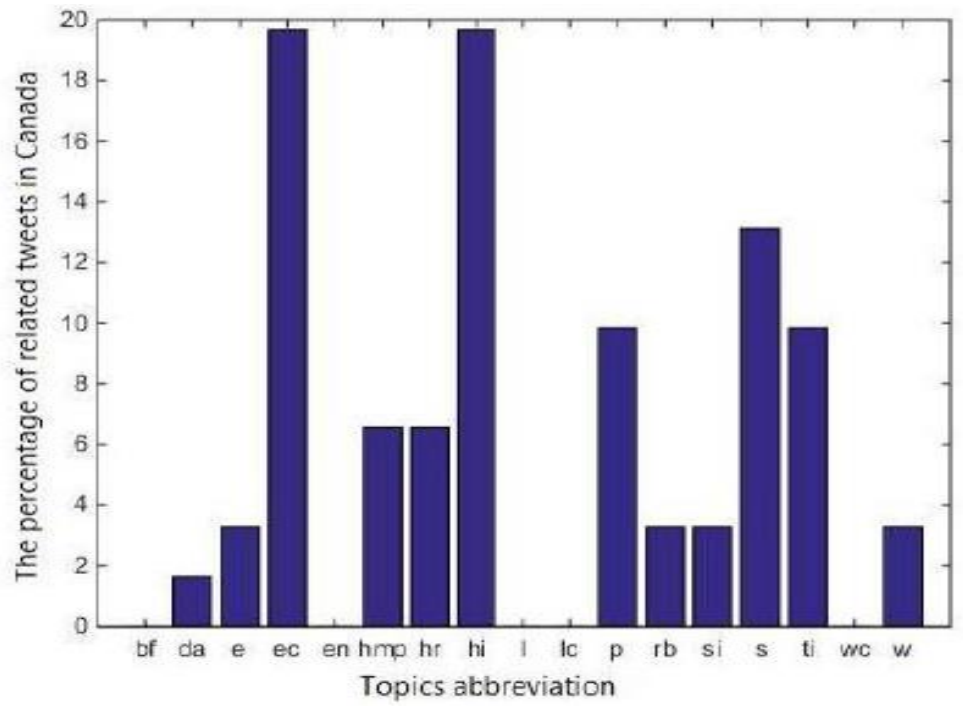

Fig. 15 Percentage of Canadian users interested in various topics.

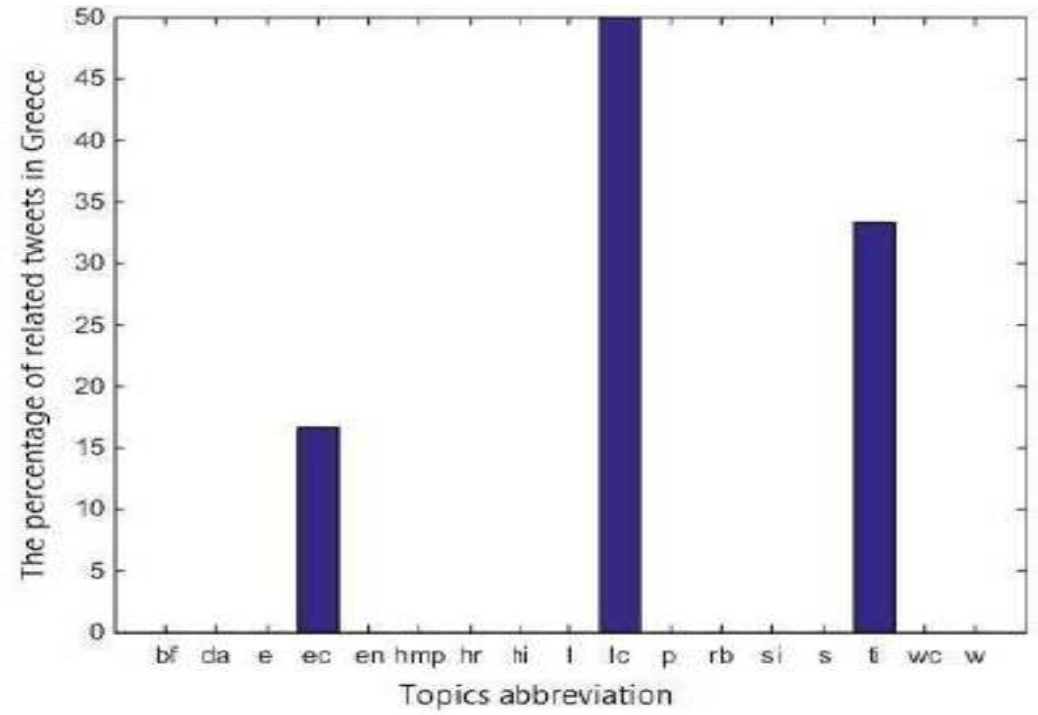

Fig. 16 Percentage of Greek users' interest in various topics. 


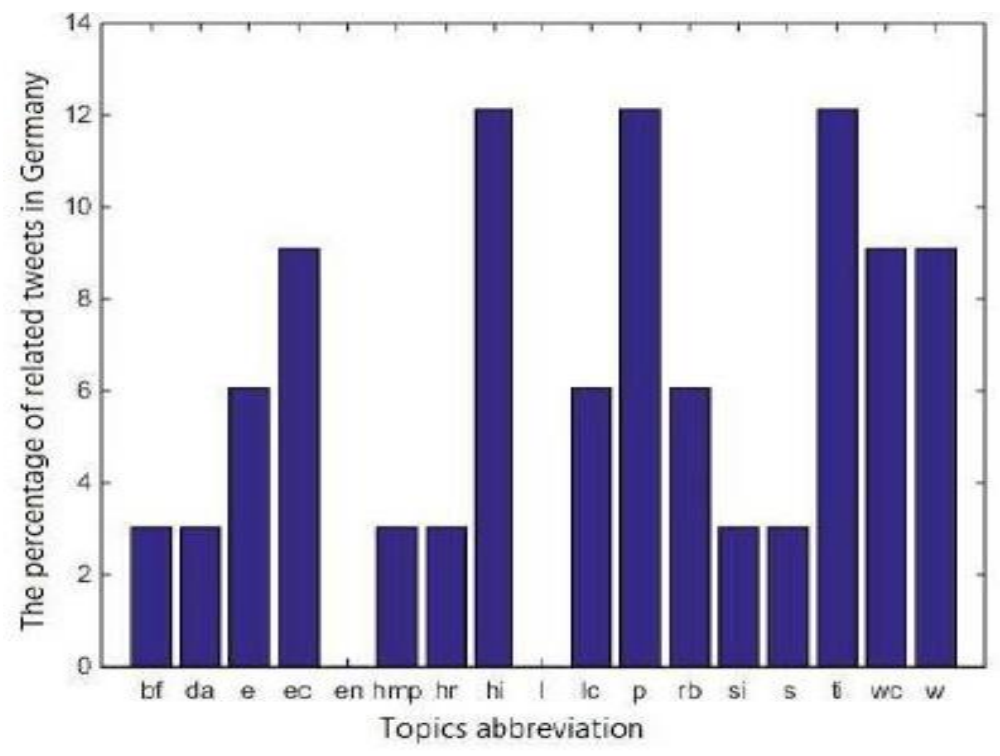

Fig. 17 Percentage of German users' interest in various topics.

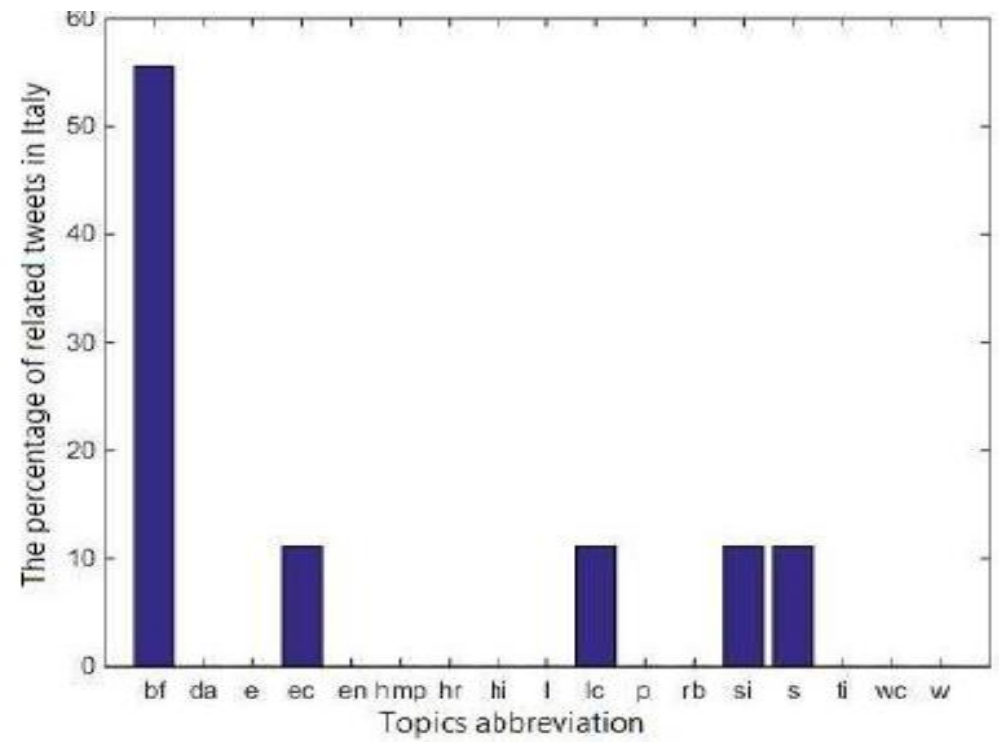

Fig. 18 Percentage of Italian users' interest in various topics. 


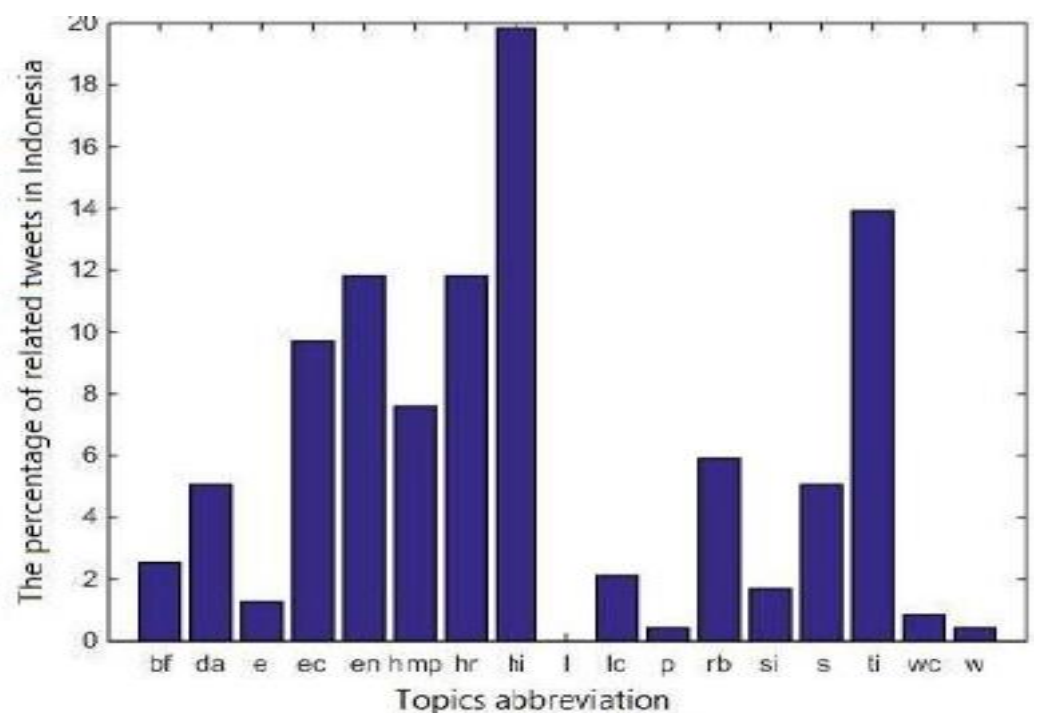

Fig. 19 Percentage of Indonesian users interested in various topics.

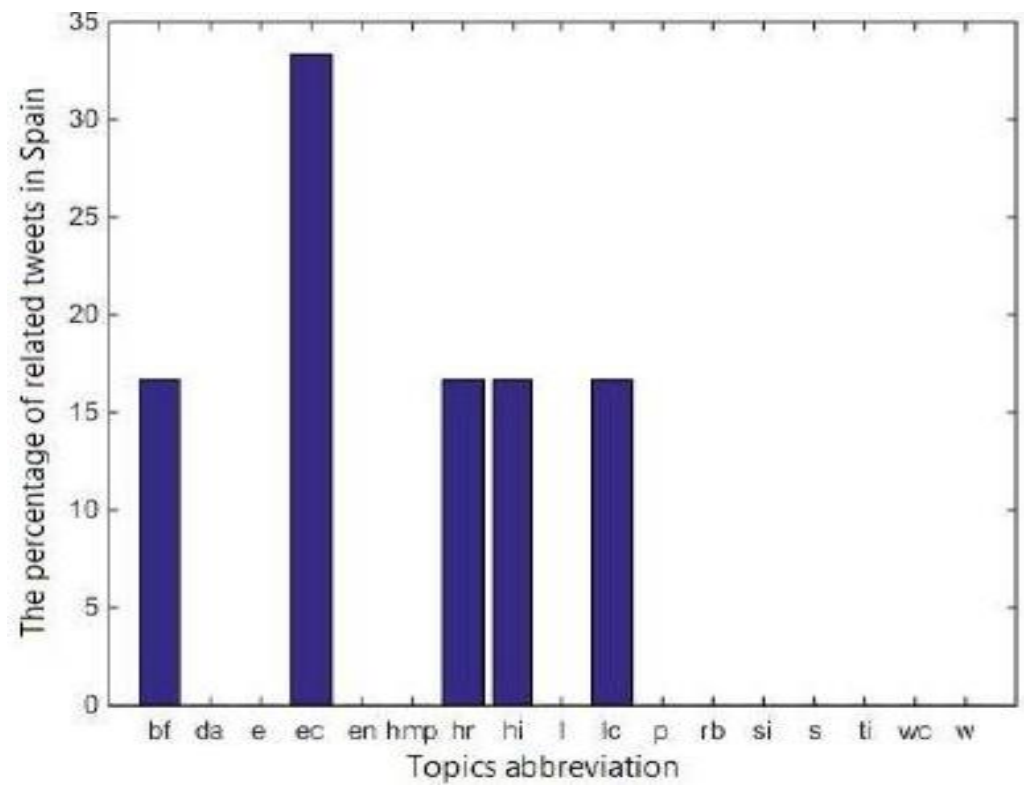

Fig. 20 Percentage of Spanish users' interest in various topics. 


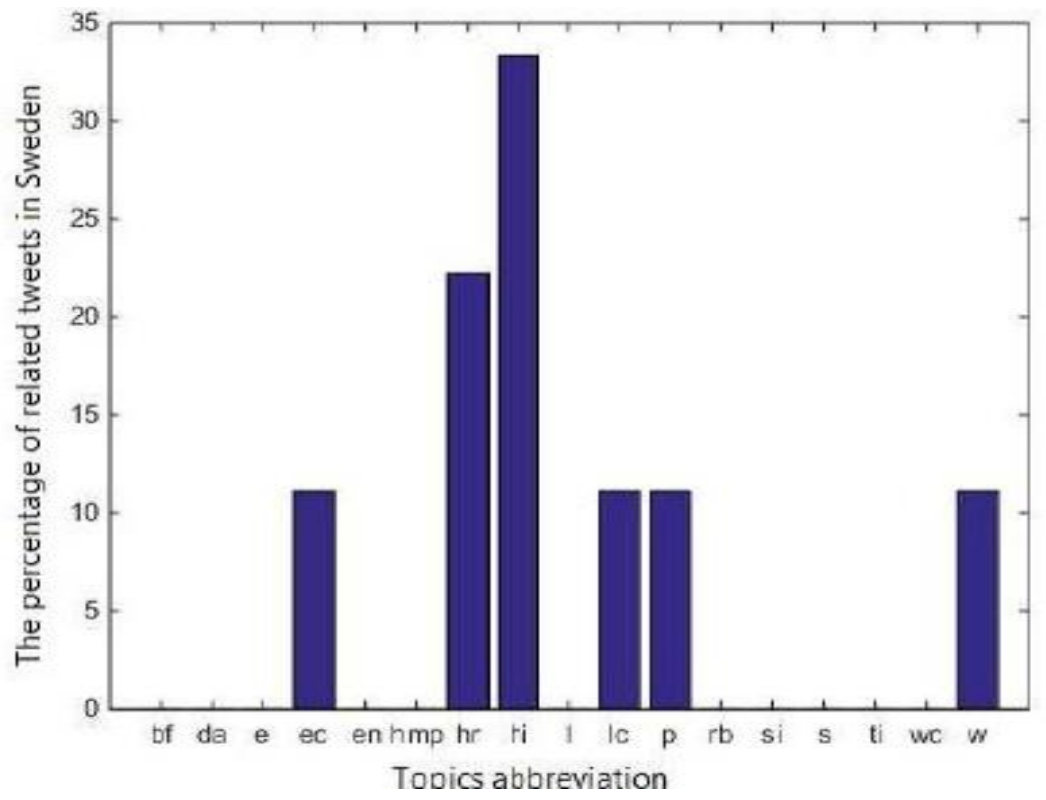

Fig. 21 Percentage of Swedish users' interest in various topics.

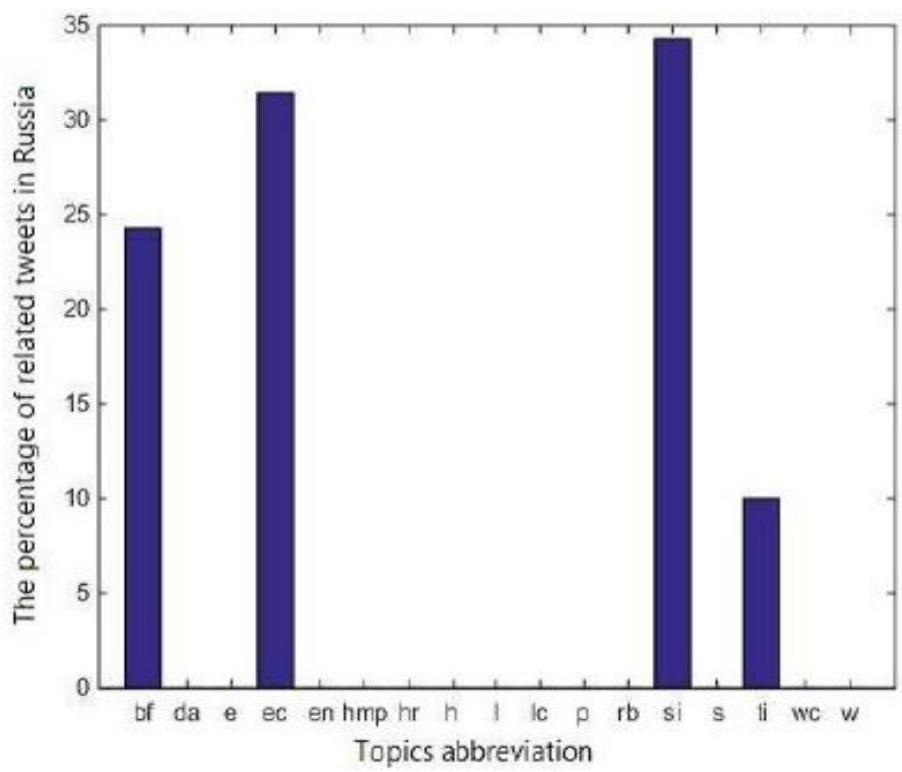

Fig. 22 Percentage of Russian users' interest in various topics. 


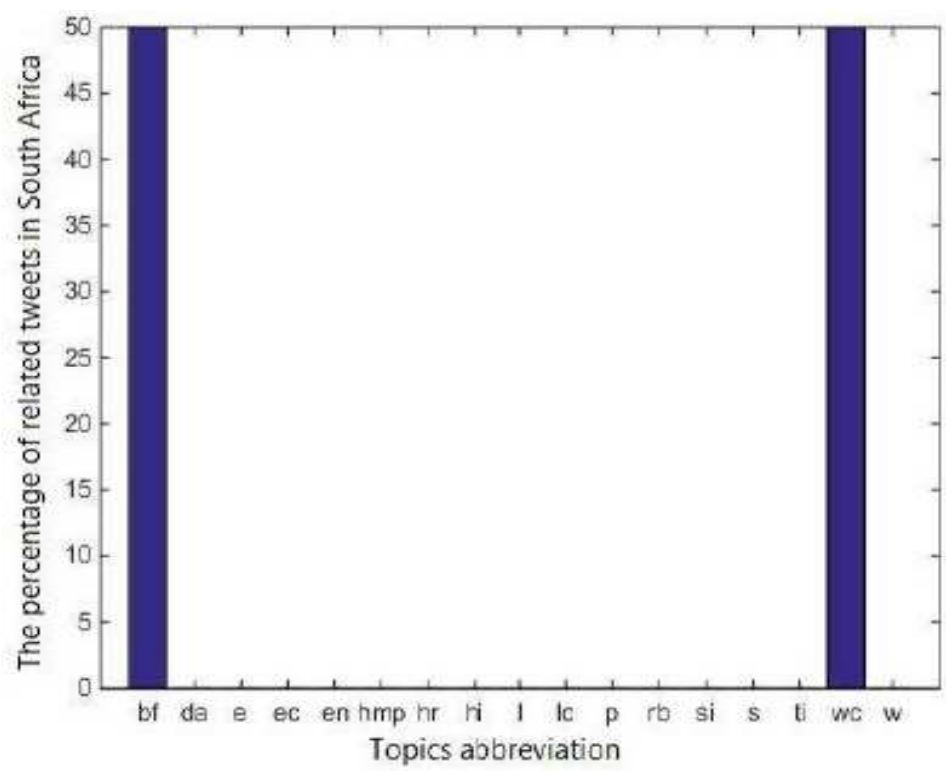

Fig. 23 Percentage of South African users interested in various topics.

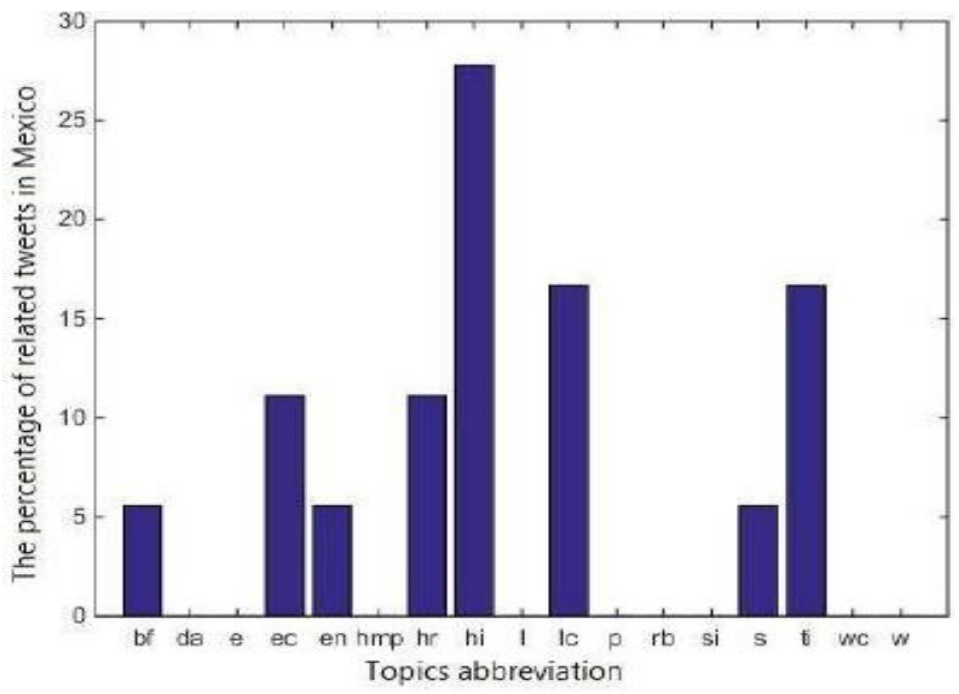

Fig. 24 Percentage of Mexican users interested in various topics. 


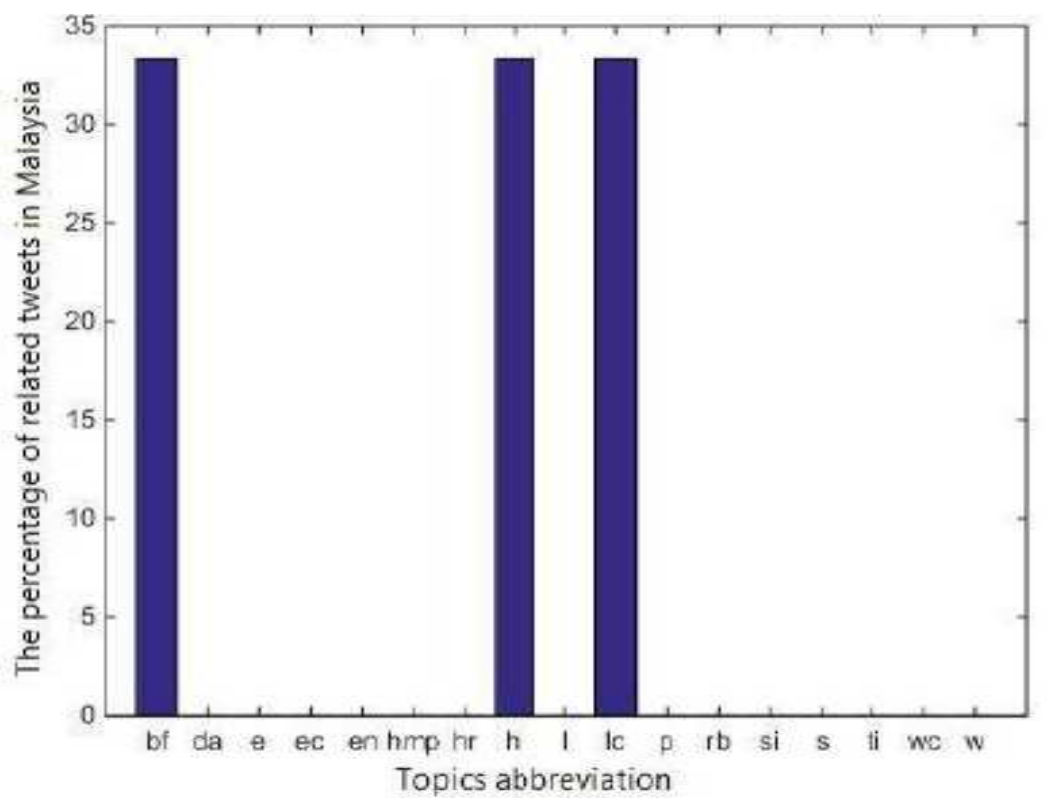

Fig. 25 Percentage of Malaysian users interested in various topics.

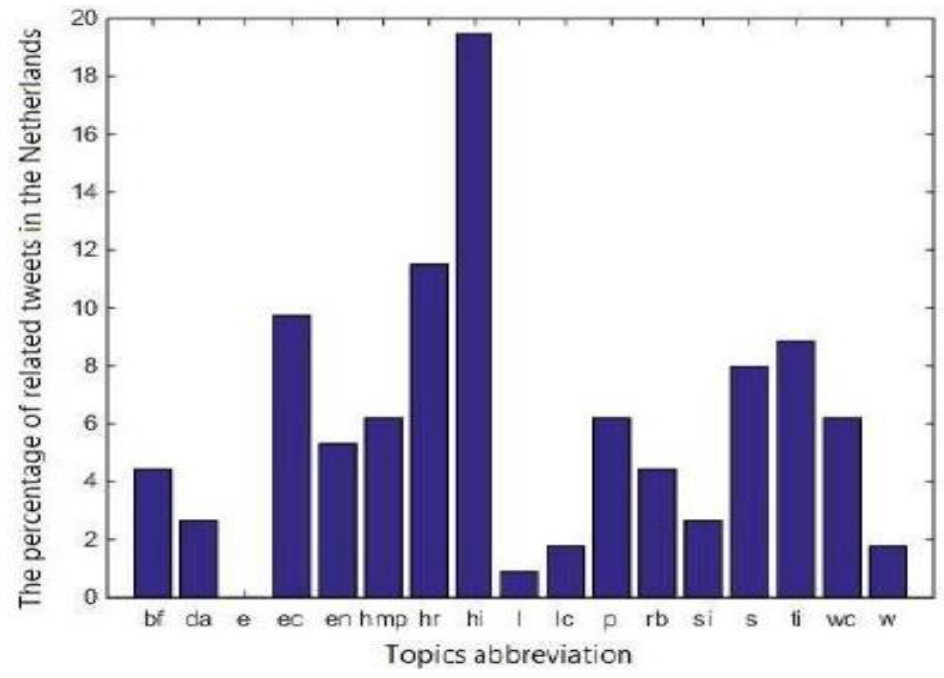

Fig. 26 Percentage of Dutch users' interest in various topics. 


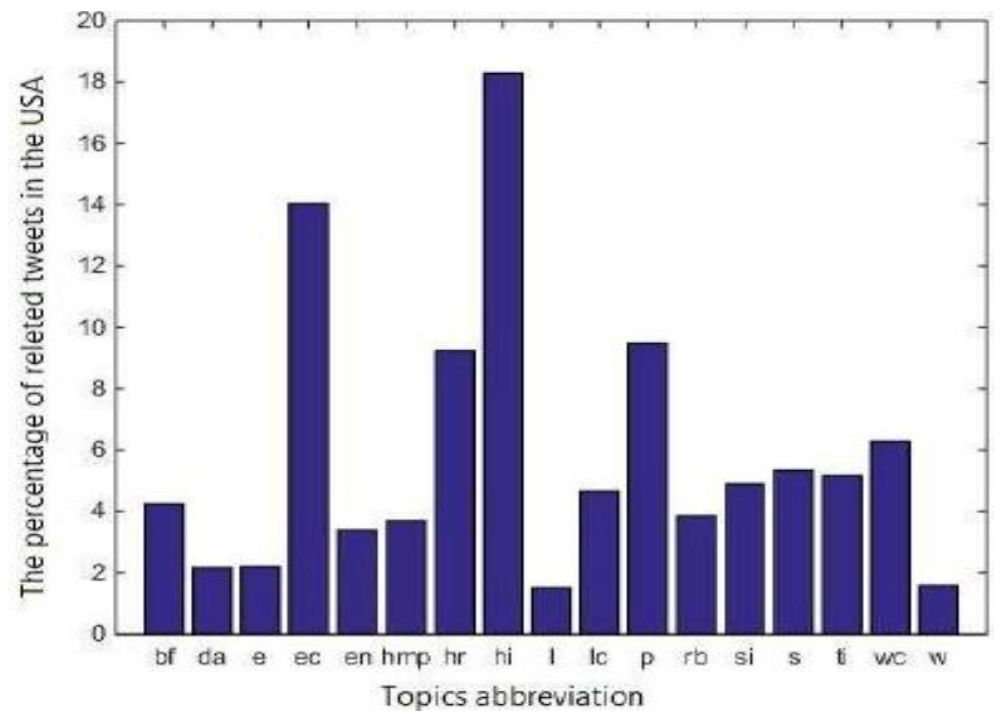

Fig. 27 Percentage of US users interested in various topics.

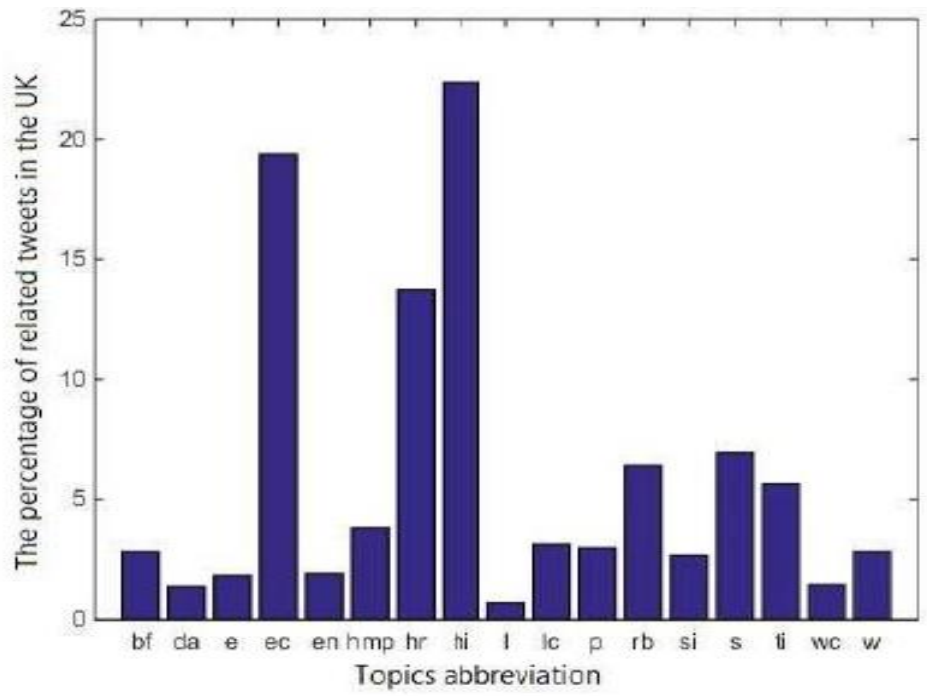

Fig. 28 Percentage of UK users interested in various topics. 


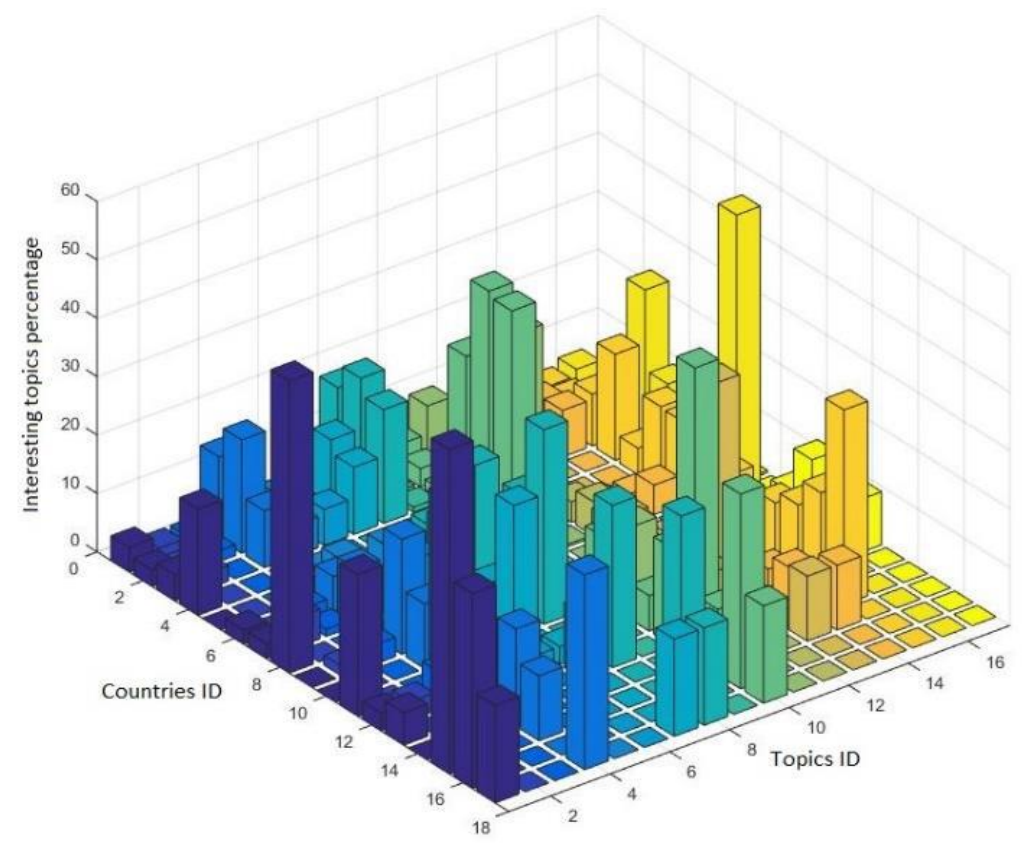

Fig. 29 Percentage of users in different countries interested in different topics.

Table. 2 Each number in the horizontal axis of the graphs represents a subject. In this table, their equivalent names are presented.

\begin{tabular}{|c|c|}
\hline ID & Topic Name \\
\hline 0 & Business_Finance \\
\hline 1 & Disaster_Accident \\
\hline 2 & Education \\
\hline 3 & Entertainment_Culture \\
\hline 4 & Environment \\
\hline 5 & Health_Medical_Pharma \\
\hline 6 & Hospitality_Recreation \\
\hline 7 & Human Interest \\
\hline 8 & Labor \\
\hline 9 & Law_Crime \\
\hline 10 & Politics \\
\hline 11 & Religion_Belief \\
\hline 12 & Social Issues \\
\hline 13 & Sports \\
\hline 14 & Technology_Internet \\
\hline 15 & War_Conflict \\
\hline 16 & Weather \\
\hline
\end{tabular}


Table. 3 Each number in the third axis of the 3D graph represents a country. In this table, their equivalent names are presented.

\begin{tabular}{|l|l|}
\hline Country ID & Country Name \\
\hline 1 & United States \\
\hline 2 & United Kingdom \\
\hline 3 & The Netherlands \\
\hline 4 & Belgium \\
\hline 5 & Denmark \\
\hline 6 & Brazil \\
\hline 7 & Indonesia \\
\hline 8 & South Africa \\
\hline 9 & Canada \\
\hline 10 & Sweden \\
\hline 11 & Russia \\
\hline 12 & Germany \\
\hline 13 & Mexico \\
\hline 14 & Greece \\
\hline 15 & Italy \\
\hline 16 & Malaysia \\
\hline 17 & Spain \\
\hline & \\
\hline
\end{tabular}

\section{Conclusion}

This paper presents a news recommendation system using the twitter social network based on the geographical location of its users. To implement and test the proposed system, a data set of 120,000 tweets, presented in paper [8] and used by the Thomson Reuters Center and topic discovery, was used. One part of this data group was used as an educational data group and the other part was used as an experimental data group. It should be noted that although the volume of the tweet data group seems small, but due to the many calculations and searches that are done on each word and phrase, the computational time is very high and in this regard in the field the data is large, so the Spark framework has been used to improve this computational time. This paper has worked on news suggestion systems, so we needed to discover the topic so that we could find the topics of interest to users based on their situation. To do this, two computational phases were designed; First, a metric was used to determine the importance of each word or phrase, as a weight for each in the relevant topic, in each specific class in the educational data group. These calculated weights were considered as a feature for each Topic class. The second phase involves calculating the degree to which a tweet relates to a particular class in the experimental data group. In order to find the relevant topics, it should be noted that for each tweet, at least one topic and a maximum of seventeen topics can be found.

Implementation was performed with 3 models of experimental and educational data group (10fold, 5-fold, 3-fold). In the first case, $10 \%$ of the whole data group is responsible for teaching the algorithm and $90 \%$ of it was used for testing. In this case, the average was $68.4518 \%$. In the second case, educational and experimental data groups included $20 \%$ and $80 \%$ of the data group, 
respectively. In this implementation, the accuracy of the answers was $74,117 \%$. In the third case, approximately $33 \%$ formed the educational data group and $67 \%$ formed the experimental data group. In this case, the accuracy rate reached $76.5163 \%$. From the point of view of this research group, the second case was considered the best case, because the accuracy of the turn increased to the first case and the third case resulted in a smaller educational data group, although the focus of this paper was on Twitter social network data. But the proposed approach can be applied to other social networks as well. It should be noted that the implementation of this paper was done on one device. With the implementation in the form of clustering, the efficiency of the system will definitely increase. Or in the first phase, when calculating the TF-IDF, only one device is used because those parts of the algorithm are not independent of each other and cannot be run in parallel.

\section{Conflict of Interest}

The authors declare that they have no conflict of interest.

\section{Declaration}

Funding: No funds, grants, or other supports was received.

Availability of data and materials: Not applicable

Ethical approval: Not applicable

Consent to participate: Not applicable

\section{Reference}

1. Zhu, Q., Zhou, X., Song, Z., Tan, J., \& Guo, L. (2019, July). Dan: Deep attention neural network for news recommendation. In Proceedings of the AAAI Conference on Artificial Intelligence (Vol. 33, No. 01, pp. 5973-5980).

2. Li, L., Zheng, L., Yang, F., \& Li, T. (2014). Modeling and broadening temporal user interest in personalized news recommendation. Expert Systems with Applications, 41(7), 3168-3177.

3. Cui, L., \& Shi, Y. (2014). A Method based on One-class SVM for News Recommendation. Procedia Computer Science, 31, 281-290.

4. Wu, C., Wu, F., An, M., Huang, J., Huang, Y., \& Xie, X. (2019, July). NPA: neural news recommendation with personalized attention. In Proceedings of the 25th ACM SIGKDD International Conference on Knowledge Discovery \& Data Mining (pp. 2576-2584).

5. Zihayat, M., Ayanso, A., Zhao, X., Davoudi, H., \& An, A. (2019). A utility-based news recommendation system. Decision Support Systems, 117, 14-27.

6. Oard, D. W. (1997). The state of the art in text filtering. User modeling and user-adapted interaction, 7(3), 141-178.

7. Sebastiani, F. (2002). Machine learning in automated text categorization. ACM computing surveys (CSUR), 34(1), 1-47.

8. Banerjee, S., Ramanathan, K., \& Gupta, A. (2007, July). Clustering short texts using wikipedia. In Proceedings of the 30th annual international ACM SIGIR conference on Research and development in information retrieval (pp. 787-788). 
9. Schönhofen, P. (2009). Identifying document topics using the Wikipedia category network. Web Intelligence and Agent Systems: An International Journal, 7(2), 195-207.

10. Haiyan, C. (2015). Measuring semantic similarity between words using web search engines. Computer Science, 42(2), 261-267.

11. Phan, X. H., Nguyen, L. M., \& Horiguchi, S. (2008, April). Learning to classify short and sparse text \& web with hidden topics from large-scale data collections. In Proceedings of the 17th international conference on World Wide Web (pp. 91-100).

12. Blei, D. M., Ng, A. Y., \& Jordan, M. I. (2003). Latent dirichlet allocation. the Journal of machine Learning research, 3, 993-1022.

13. Mihalcea, R., Corley, C., \& Strapparava, C. (2006, July). Corpus-based and knowledge-based measures of text semantic similarity. In Aaai (Vol. 6, No. 2006, pp. 775-780).

14. Zhang, H., Yen, J., Giles, C. L., Mombaster, B., Spiliopoulou, M., Srivastava, J., ... \& McCallum, A. (2007). WebKDD/SNAKDD 2007: web mining and social network analysis post-workshop report. Acm Sigkdd Explorations Newsletter, 9(2), 87-92.

15. Zhao, D., \& Rosson, M. B. (2009, May). How and why people Twitter: the role that microblogging plays in informal communication at work. In Proceedings of the ACM 2009 international conference on Supporting group work (pp. 243-252).

16. Shamma, D. A., Kennedy, L., \& Churchill, E. F. (2009, October). Tweet the debates: understanding community annotation of uncollected sources. In Proceedings of the first SIGMM workshop on Social media (pp. 3-10).

17. Shamma, D., Kennedy, L., \& Churchill, E. (2010). Tweetgeist: Can the twitter timeline reveal the structure of broadcast events. CSCW Horizons, 26.

18. Angel, A., Koudas, N., Sarkas, N., \& Srivastava, D. (2009, June). What's on the grapevine?. In Proceedings of the 2009 ACM SIGMOD International Conference on Management of data (pp. 10471050).

19. Bansal, N., \& Koudas, N. (2007, September). Blogscope: a system for online analysis of high volume text streams. In Proceedings of the 33rd international conference on Very large data bases (pp. 1410-1413).

20. Sankaranarayanan, J., Samet, H., Teitler, B. E., Lieberman, M. D., \& Sperling, J. (2009, November). Twitterstand: news in tweets. In Proceedings of the 17th acm sigspatial international conference on advances in geographic information systems (pp. 42-51).

21. Dong, A., Zhang, R., Kolari, P., Bai, J., Diaz, F., Chang, Y., ... \& Zha, H. (2010, April). Time is of the essence: improving recency ranking using twitter data. In Proceedings of the 19th international conference on World wide web (pp. 331-340).

22. Dong, A., Chang, Y., Zheng, Z., Mishne, G., Bai, J., Zhang, R., .. \& Diaz, F. (2010, February). Towards recency ranking in web search. In Proceedings of the third ACM international conference on Web search and data mining (pp. 11-20).

23. Agrawal, D., Bernstein, P., Bertino, E., Davidson, S., Dayal, U., Franklin, M., ... \& Widom, J. (2011). Challenges and opportunities with Big Data 2011-1.

24. Pentland, A. (2012). Reinventing society in the wake of big data. Edge, 30 August 2012. URL: http://www. edge. org/conversation/reinventing-society-inthe-wake-ofbig-data (date of access: 28.01. 2013).

25. Lohr, S. (2012). The age of big data. The New York Time.

26. Jacob, W. (2014). Book Review: Viktor Mayer-Schonberger and Kenneth Cukier, Big Data: A Revolution That Will Transform How We Live, Work and Think.

27. Manovich, L. (2011). Trending: The promises and the challenges of big social data. Debates in the digital humanities, 2(1), 460-475. 
28. Boyd, D., \& Crawford, K. (2011, September). Six provocations for big data. In A decade in internet time: Symposium on the dynamics of the internet and society.

29. Hu, L., Li, C., Shi, C., Yang, C., \& Shao, C. (2020). Graph neural news recommendation with long-term and short-term interest modeling. Information Processing \& Management, 57(2), 102142. 